\title{
Characterisation and improvement of the quality of mixing of recycled thermoplastic composites
}

\author{
Guillaume A. Vincent ${ }^{\mathrm{a}, \mathrm{b}}$, Thomas A. de Bruijn ${ }^{\mathrm{c}}$, Sebastiaan Wijskamp ${ }^{\mathrm{b}}$, \\ Mohammed Iqbal Abdul Rasheed ${ }^{a}$, Martin van Drongelen ${ }^{\mathrm{a}, *}$, Remko Akkerman ${ }^{\mathrm{a}}$ \\ ${ }^{a}$ Chair of Production Technology, University of Twente, Enschede 7522 NB, the Netherlands \\ ${ }^{\mathrm{b}}$ ThermoPlastic composites Research Center, Palatijn 15, Enschede 7521 PN, the Netherlands \\ ${ }^{\mathrm{c}}$ ThermoPlastic composites Application Center, Saxion University of Applied Sciences, M.H. Tromplaan 28, Enschede 7513 AB, the Netherlands
}

\section{A R T I C L E I N F O}

\section{Keywords:}

Long fibre

Thermoplastic composites

Mixing

Spatial statistics

Image analysis

\begin{abstract}
A B S T R A C T
A low-shear mixer was used to blend thermoplastic composite scrap material into a molten mixed dough, which was then compression moulded. This process is a key step in a novel recycling solution for thermoplastic composites. A study was carried out to characterise the quality of mixing (QoM) of the blended doughs to understand how to improve the QoM of mixed doughs towards further improvement and implementation of the recycling solution. In order to achieve this, the effect of mixing parameters and fibre length on the QoM were studied. This study used shredded C/PPS flakes, originating from consolidated laminate scrap. These flakes are about $20 \mathrm{~mm}$ in size, and contain woven fabric reinforcement, making them far different from regular pellets, and therefore more difficult to mix. The QoM was characterised by means of image analysis of a large set of cross-sectional microscopy images, based on which the scale and intensity of segregation of the fibre clusters were evaluated. Bundle size distribution was determined by applying Delaunay triangulations to cluster the fibre centres. These methods were found to be suitable for characterising the QoM of such doughs. Increasing the mixing time and mixing speed were identified as key ways to improve the mixing process. With the current mixing machine, it is also suggested not to use fibres longer than $15 \mathrm{~mm}$ on average in order to limit intra-dough variability. For doughs made of fibres longer than $15 \mathrm{~mm}$, improvements on the mixing device could sufficiently increase the QoM.
\end{abstract}

\section{Introduction}

The application of long fibre thermoplastics (LFTs) attracted considerable interest in the past decades $[1,2]$. On one hand, they offer and allow for an increased geometrical complexity, from a design point of view, compared to continuous fibre composites. On the other hand, they exhibit mechanical properties intermediate to short and continuous fibre composites. Several categories of LFTs have been brought to market including thermoplastic bulk moulding compounds [3,4], glass mat thermoplastics and LFT pellets $[2,5,6]$. During the same period, the production and demand for continuous fibre thermoplastic composites (TPCs) has increased considerably, leading to a rise in TPC production scrap. The economic benefits of recycling this scrap, in conjunction with environmental and legislative incentives, have encouraged the development of recycling solutions for TPCs. Recently, both De Bruijn et al. [7,8] and Janney et al. [9] separately developed a recycling route for TPC scrap inspired by the processing of LFT pellets. A schematic view of the recycling solution implemented by De Bruijn is shown in Fig. 1. Production offcuts, such as trims and nesting scrap, are first collected and shredded into flakes. Most often, these flakes are multi-layered material and may consist of woven fabric, as in $[8,10]$ and as shown in Fig. 1. Flakes are then processed in a blender to form a molten mixed dough that is transferred directly to a press for compression moulding. The mixing phase used in this study is similar to the mixing of LFT pellets. The pellets or flakes are processed in a device that melts the polymer and disperses the fibres. However, mixing of flakes described here can be substantially more difficult than mixing LFT pellets that have been designed to disperse easily [5].

The subsequent processing step, after mixing, involves squeezing the mixed dough into a mould. Its flow behaviour depends on the amount of fibres, fibre length, the interaction of fibre bundles in the dough, and thus the dispersion of bundles. Once a component has been moulded, its mechanical properties are also linked to fibre length, orientation and the dispersion of fibre bundles. A previous study by De Bruijn [7] showed that mixing the multi-layered TPC flakes significantly improves the mechanical properties of the moulded panels, compared to compression

\footnotetext{
* Corresponding author.

E-mail address: m.vandrongelen@utwente.nl (M. van Drongelen).
} 

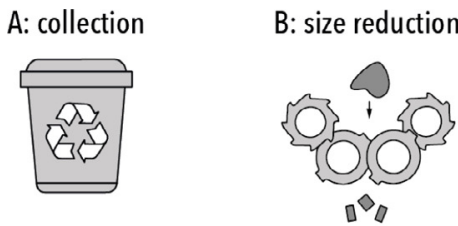

$$
\text { C: mixing }
$$
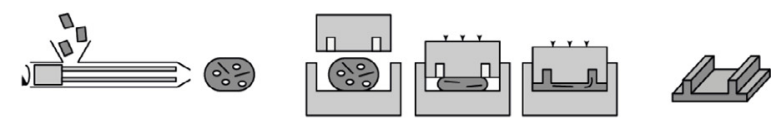

E: release recycling solution. The top row illustrates the various processing steps while the bottom row shows the material state between each step.

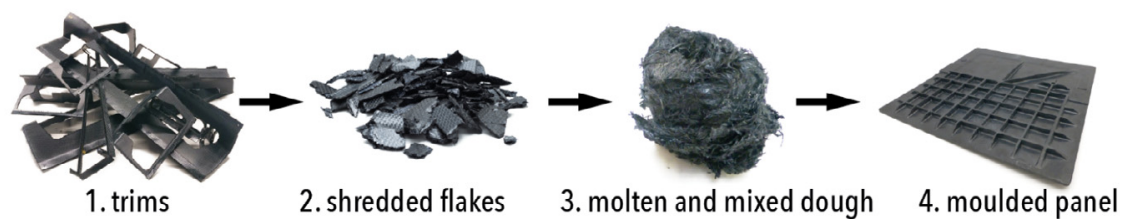

moulding of non-mixed flakes. This improvement is mainly driven by the micro-structural change: from stacked, thick flakes to homogeneously entangled fibre bundles. Additionally, the recycling solution presented here prevents fibre attrition during mixing by using a low-shear mixer, in order to limit the reduction in mechanical properties, which depend on the fibre length. The mixing of flakes, and the resulting micro-structure, is therefore crucial for the recycling process. Consequently, a proper understanding of the mixing step is required, as well as a good definition of the quality of mixing (QoM) of mixed flakes. A suitable characterisation method for QoM must be developed and implemented.

The present article will deal with the mixing step in the recycling solution developed by De Bruijn et al. and characterise the QoM of the mixed material, with the objective of understanding how to improve the QoM of mixed doughs. In order to achieve this, several aspects of the QoM that can be applied to fibre reinforced composites will be selected from literature, improved and implemented. Finally, the effects of mixing settings and fibre length on QoM will be studied.

\section{Literature review}

The study of mixing and segregation is a field of spatial statistics that, over the past decades, has been widely applied to various fields of science, ranging from ecology to the material blending [11-13]. A first comprehensive definition of QoM was proposed by Danckwerts in 1952 [14] by introducing two aspects: the intensity of segregation (IoS) and the scale of segregation (SoS). The IoS corresponds to the coefficient of variation $(\mathrm{CoV})$ of the concentration of species in the system, also known as evenness [15]. With regard to blending fibre composites, it refers to the $\mathrm{CoV}$ of the local fibre fraction. The SoS represents a characteristic length of segregation, or clustering, in the system. SoS represents, for instance the maximum striation thickness for mixed fluids, or the size of fibre clusters for fibre composites. Fig. 2 shows a visual representation of good and poor IoS and SoS, with each sub-figure representing a cross-sectional micrograph of fibre composites. The studies that followed Danckwerts extended his initial work and defined other aspects of segregation [15]. Exposure corresponds to the rate of change in segregation, which has some importance along the length of screw extruders, for instance. Density is a measure of the mass or volume of one of the constituents per unit volume or area. Lastly, centralisation corresponds to the aggregation of a constituent away from its centre.

The first two aspects of segregation were continuously measured in various fields related to spatial statistics [11-13] by adapting the measurement methods for IoS and SoS to specific situations. IoS and SoS are particularly well-suited for the mixing of discontinuous-fibre composites and have been studied by various authors [13,16-29]. The latter three aspects of segregation - namely exposure, density and centralisation have been scarcely used for fibre composites and polymer blends.

The problem presented in this paper is similar, in a way, to other mixing problems for discontinuous-fibre composites or polymer blends, and can be summarised as follows. Two immiscible species, fibres and polymer, are initially highly segregated in the form of large clusters of flakes (large multi-layered flakes) and polymer granulates. During mixing, the flake structure delaminates and disentangles into loose bundles, eventually leading to a more even distribution of fibres in the dough. Measuring IoS and SoS only requires a snapshot of the final state of the dough to be measured, while the exposure can be difficult to determine for mixed discontinuous-fibre composites because it is usually only possible to capture the final state. Regarding the centralisation, Kukukova et al. [15] state that 'it does not add information about the mixing problem beyond the scale and intensity of segregation'. However, poor mixing of fibre bundles can lead to a situation in which they are locally well dispersed (good IoS and SoS), but accumulate in some areas.

The characterisation of the QoM of fibre-reinforced plastics is diverse. Most research is based on image analysis of cross-sectional microscopy. Several methods have been implemented and can be categorised as follows.

Analyses based on quadrats

Several authors worked with cross-sectional microscopy of fibre composites divided into square grids of various sizes. The fibre fraction or the presence of fibre in each cell is measured and recorded. Fig. 2(a) to 2(d) represent quadrats filled with fibres at various degrees of evenness and clustering. Inoue et al. [16] and Spowart et al. [17] measured QoM by determining a box-counting dimension of the presence of fibres in the cells. This is indirectly related to the SoS of the fibre clusters. Li et al. [18] also worked on cross-sectional micrographs of fibrereinforced concrete and measured the $\mathrm{CoV}$ of the local fibre fraction in the cells. Using the CoV of local fibre fraction is found to lead to the closest estimation of the IoS for such a system, sufficiently characterising the dispersion of fibres in the system. Normalising it to the CoV of the non-mixed material allows for the measurement of the improvement of evenness due to mixing. This is a better measure than just the $\mathrm{CoV}$ for comparing mixers or for identifying how various materials behave in a mixer. Besides, the variation in CoV per cell size has scarcely been studied, and may lead to novel results.

Analyses based on microstructural features

Le Baillif et al. [19] compounded cellulose fibre reinforced polypropylene using a twin screw extruder and characterised the dispersion of fibres using cross-sectional microscopy. They measured the distribution of the size of the fibre clusters, which is an estimation of the SoS. Yazdanbakhsh et al. [13] formulated a new definition of particle dispersion, dispersive work, as the minimum amount of work per domain to move all particles to a uniform state of equally distant particles. It is therefore based on the distribution of the particle locations in the domain. Wang et al. [20] mixed carbon fibre reinforced cement and measured the dispersion of fibres with the aid of 3D tomographic images. A dispersion coefficient was defined as the $\mathrm{CoV}$ of the size of fibre clusters.

Various researchers determined fibre dispersion by either considering the size of agglomerates or their CoV. The former seems to be a good measure of the SoS, whereas the latter characterises the poly-dispersity 


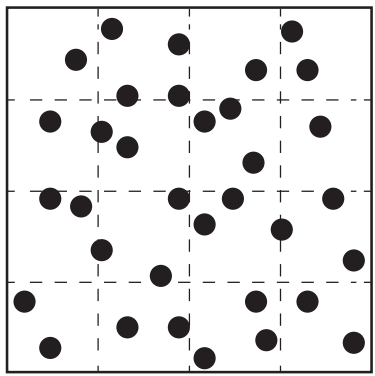

(a) $\operatorname{good} I o S, \operatorname{good} S o S$

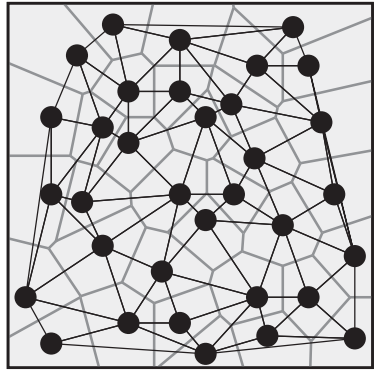

(e) $\operatorname{good} I o S, \operatorname{good} S o S$

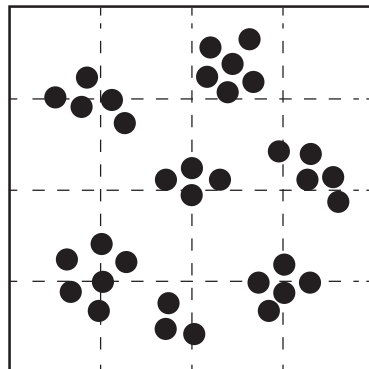

(b) good IoS, poor $S o S$

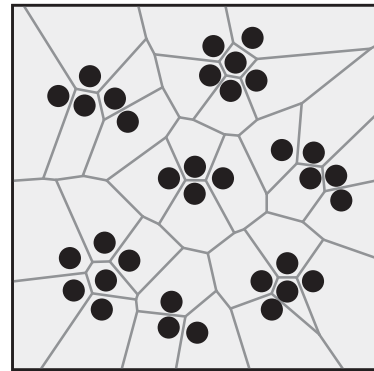

(f) $\operatorname{good} I o S$, poor $S o S$

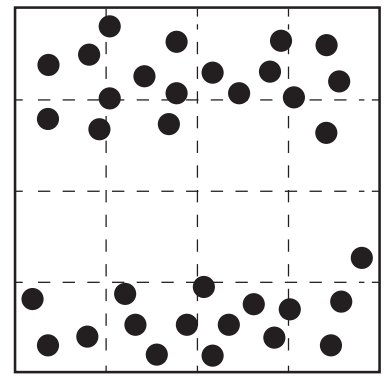

(c) poor IoS, good $S o S$

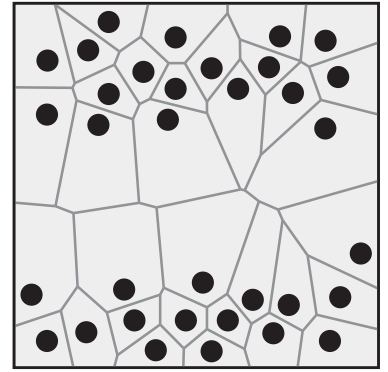

(g) poor IoS, good $S o S$

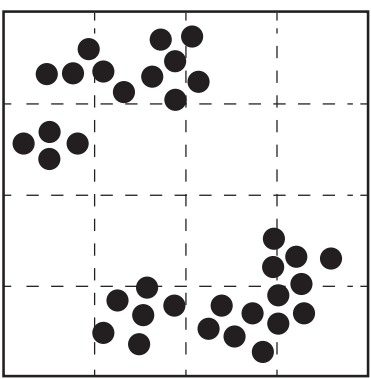

(d) poor IoS, poor $S o S$

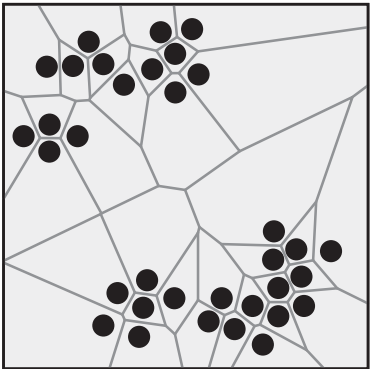

(h) poor IoS, poor $S o S$

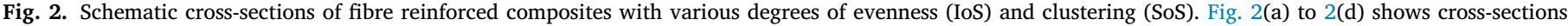

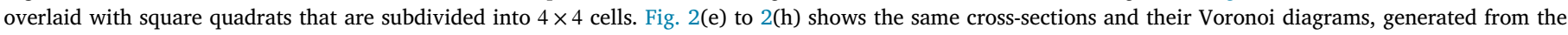
centre of the fibres. The dual graph of the Voronoi diagram, the Delaunay tessellation, is only shown in Fig. 2(e) for clarity.

of fibre clusters in a blend but hardly indicates the SoS, or any aspect of QoM defined in [15]. Dispersive work, as defined by Yazdanbakhsh et al., may be a good measure of either clustering, evenness or centralisation in some situations. Nonetheless, it is not capable of distinguishing systems made up of large clusters from those with poor centralisation.

Analyses based on Delaunay triangulation

Several researchers characterised the QoM of discontinuous-fibre composites based on photographs (made by optical or electron microscopes) using the Delaunay triangulation or its dual graph, the Voronoi diagram [21-26]. Voronoi diagrams are constructed on the centroids of the particles, from which several statistical quantities are measured. Fig. 2(e) to 2(h) shows the Voronoi diagrams of four schematic crosssections of fibre composites, with various levels of IoS and SoS. The figures highlight the differences in Voronoi cell sizes and cell distributions for these four situations. In most cases, the distribution of the areas of the Voronoi cells was used. Additionally, the distribution of the mean near-neighbour distance, which can be output from the Delaunay triangulation (see Fig. 2(c)), was computed. It was found that the $\mathrm{CoV}$ of the mean near-neighbour distance or the $\mathrm{CoV}$ of the Voronoi cell sizes are a powerful tool to characterise the homogeneity of the samples. However, such a measure hardly differentiates between the evenness of particle dispersion (IoS) and their clustering (SoS). Considering two systems, one with poor evenness and a second highly clustered, one can find many fibres with both close and distant neighbours, leading to a similar measure (see Fig. 2(g) and 2(f)). Additionally, in all aforementioned studies the mean near-neighbour distance was measured as the Euclidean distance between fibre centroids in 2D microscopic images. Discontinuous cylindrical fibres can orient themselves in any direction in a composite specimen, which means that a cross-sectional micrograph of such a specimen shows fibres as ellipses of various eccentricities and orientations. The actual distance between fibres, however, is the distance between their centrelines, which depends on the ellipse parameters when cut in a 2D plane. Therefore, the Euclidean distance between fibre centroids leads to interpretation errors if the ellipses' eccentricities and orientations are not taken into account.

Analyses based on physical measurements
Several studies on discontinuous-fibre reinforced composites characterised QoM by performing global physical measurements. Yang et al. [27] and Ozyurt et al. [28] worked with carbon-fibre-reinforced cement and measured the electrical resistance of their specimens to determine fibre dispersion. Similarly, Yenjaichon et al. [29] used electrical resistance tomography to determine the QoM of kraft pulp suspensions. Although these measurement methods can be non-destructive, they struggle to distinguish the various aspects of segregation, in particular IoS and SoS.

Outcome

The previous studies have sought to tackle the problem of characterising the QoM of fibre reinforced plastics with various methods[13,16-29], mainly based on image analysis of microscopy images. Most of these methods were inspired by a large corpus on spatial statistics $[12,14,15,30,31]$, but the methods implemented for fibre composites often lack the mathematical rigour of the latter. Especially, most fail to distinguish the evenness of particle dispersion from fibre clustering. It is crucial to study both IoS and SoS.

Cross-sectional microscopy appears to be a relevant method of characterising the QoM of discontinuous fibre composites. The stochastic nature of these materials requires a considerable number of microscopic photographs to provide statistically satisfactory results. The CoV of the local fibre fraction is found to be a pertinent measure of IoS, which represents the evenness of a sample. However, the CoV characterises the material, not the mixing technology, which is why it should be normalised to the CoV of the local FVFs in the initially non-mixed material to measure the improvement in evenness thanks to the mixing step. This measurement method was introduced by Danckwerts [14] and later used for various mixing problems [15]. The normalised $\mathrm{CoV}$ appears to be a better measure to compare different materials after mixing, or different mixing systems, and it will therefore be examined in this study. Close attention will also be paid to the effect of cell size on IoS, since, to the knowledge of the authors, it has not been reported in relevant literature. No previously investigated measure of SoS seemed adequate for fibrereinforced composites, which is why one close to the general definition mentioned in Kukukova et al. [15] will be implemented. 


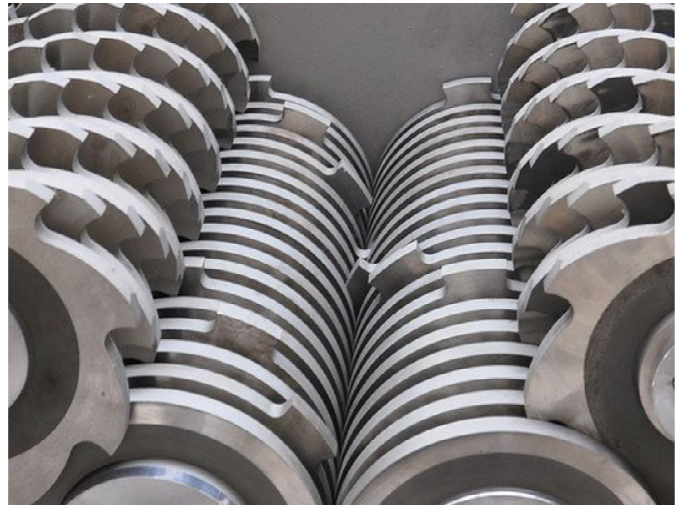

Fig. 3. Inside view of a four-shaft shredder (image courtesy of UNTHA GmbH).

Boxplots of the FLDs studied in article

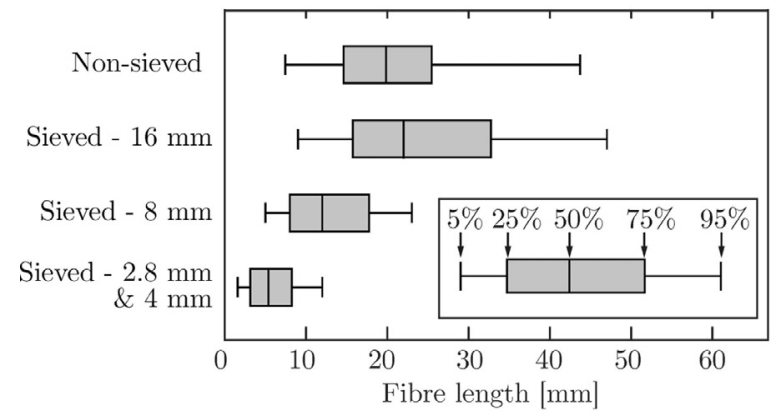

Fig. 4. FLDs of the various types of flakes used in this article.

The measurement of fibre clustering using Delaunay triangulation appears to be valuable for discontinuous-fibre composites. The exact determination of the fibre clusters might not be the prime objective when characterising QoM, but this does drive the mechanical and rheological properties of the LFTs. As of yet, little attention has been paid to correcting the distances and areas with the ellipses' orientation and eccentricity. This correction is implemented in this article to help measure the size of fibre clusters in a more precise way.

\section{Materials and methods}

\subsection{Material}

The scrap material used for this study was collected at the forming stage of various manufacturing sites. It consists of laminated TPC offcuts, made of TenCate Cetex ${ }^{\circledR}$ TC1100 5HS carbon/PPS (C/PPS) at $50 \%$ fibre volume fraction (FVF), as shown in Fig. 1 (1. trims). The carbon fabric is woven with tows that contain 3,000 fibres each. The offcuts were shredded in-house using a two-shaft shredder made by UNTHA shredding technology Gmbh, Austria (see Fig. 3). The output flakes of typically $20 \times 20 \times 3 \mathrm{~mm}$ can be seen in Fig. 1 (2. shredded flakes). Some batches of shredded flakes were sieved with a multi-stage vibrating sieve. The fibre length distribution (FLD) of the output flakes, as well as the shredding and sieving process, were previously characterised in $[10,32]$. The FLDs of the categories of flakes used in this study are shown in Fig. 4. Sieved - $16 \mathrm{~mm}$ and Sieved $-8 \mathrm{~mm}$ correspond to the flakes that first passed through the sieves with an aperture of $22.4 \mathrm{~mm}$ and $11.2 \mathrm{~mm}$, respectively, before failing to pass through the sieves with an aperture of $16 \mathrm{~mm}$ and $8 \mathrm{~mm}$, respectively. In addition Sieved $-2.8 \mathrm{~mm}$ \& $4 \mathrm{~mm}$ consists of a blend of the flakes that failed to pass through the sieves with an aperture of $2.8 \mathrm{~mm}$ and $4 \mathrm{~mm}$. C/PPS LFT pellets with $3 \mathrm{~mm}$ fibres were used to compare the QoM of the recycled material with commercially available pellets. These pellets are known as Luvo- com ${ }^{\circledR} 1301 / \mathrm{XCF} / 30$ and are produced at $30 \%$ fibre weight fraction, or approximately $24 \%$ FVF. This study also used polymer granulates to dilute the flakes or pellets during the mixing stage, namely Fortron ${ }^{\circledR} 0214$ PPS granulates by Celanese. Two other materials were used for comparison purposes:

- Short fibre pellets that were injection moulded at $40 \%$ fibre weight fraction (or around 32\% FVF) with fibres of $200 \mu \mathrm{m}$ in length.

- Single-layer woven fabric prepregs measuring $10 \times 10 \mathrm{~mm}^{2}$, which were compression moulded at $50 \%$ FVF. Note that this material is not mixed in any way. More information on the material and processes can be found in [33].

\subsection{Low-shear mixing}

After the offcuts had been shredded, the resulting flakes or pellets were fed into a low-shear mixer together with PPS granulates to dilute the fibre suspension to $20 \%$ FVF. The device was developed and manufactured at the Centre of Lightweight Structures in the Netherlands in 1998 [34]. Contrary to typical screw extruders, the low-shear mixer consists of a heated hollow cylinder with a $70 \mathrm{~mm}$ diameter, and features three eccentrically-located heated rods rotating inside. An illustration of this device is shown in Fig. 5. The three rods are placed at different distances from the axial centre of the cylinder (Fig. 5 on the right). The mixer is fed by means of a hopper located on the cylinder. After feeding, a piston pushes the material inside the cylindrical cavity, which closes the feed opening. The heated rods then start to rotate around the axial centre at a speed varying from 5 rotations per minute (rpm) to $15 \mathrm{rpm}$, for 5 to $20 \mathrm{~min}$. During this phase, the polymer starts to melt, causing the flakes to delaminate, the woven structure of the plies to loosen, and eventually the bundles to spread into clusters of various sizes. At the end of the mixing phase, the piston pushes the material out of the mixer, which is now a molten, mixed dough. During the entire mixing process, the material is not compressed and consequently only fills part of the cavity, ranging from $20 \%$ to $50 \%$. The rest is filled with air and, possibly, volatile by-products. As a result, the extruded dough is very porous, and even swells a small amount when pushed out, reaching a final diameter of $100 \mathrm{~mm}$ after ejection from the cylinder and gate, which both have a diameter of $70 \mathrm{~mm}$. In this mixing device, the rotation speeds of the low-shear mixer are typically $10 \mathrm{rpm}$ and the distance between the rotating rods and the cylindrical cavity is about $10 \mathrm{~mm}$. This results in very low shear rates compared to those found in typical screw extruders. As a consequence, fibres are less prone to breaking during the mixing phase [35]. Besides, the low-shear mixer allows for a batch-type process rather than the continuous process typical for screw extruders, as a result of which the residence time of the material is equal for all particles. The low-shear device is still in its prototype phase and has not been studied extensively. Therefore the mechanisms of mixing in such a device are still poorly known.

A fractional factorial design of experiments (DoE) of resolution IV was performed by varying the mixing speed, mixing time, cavity filling ratio and mixing temperature. Two levels were chosen for these four factors in addition to a central point. The doughs and their mixing settings are listed in Table 1. The resolution (IV) of the DoE makes it possible to identify the significance and strength of the main factors. However, the two-factor interactions for such a resolution are confounded with other two-factor interactions so they will not be analysed in order to prevent interpretation errors. The reproducibility of the mixing process was investigated by producing an extra dough for three of the DoE settings (see tests \#14, \#15, \#16 in Table 1). Some doughs were also produced with various FLDs - rows \#10 to \#13 in Table 1.

\subsection{Microscopy}

In the recycling process developed by De Bruijn et al. [7], the dough is directly transferred from the mixer to a press for compression mould- 
CAD model of the low-shear mixer

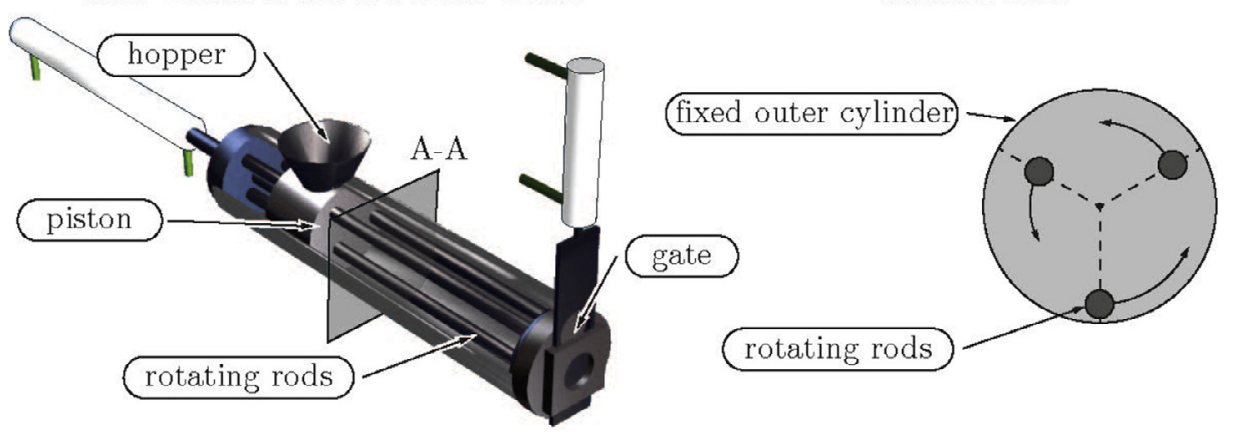

Fig. 5. A schematic of the low-shear mixing device is shown on the left. It highlights several key elements that are explained in Section 3.2. The crosssection A-A is drawn on the right-hand side of the figure.

Table 1

List of the various doughs manufactured and tested.

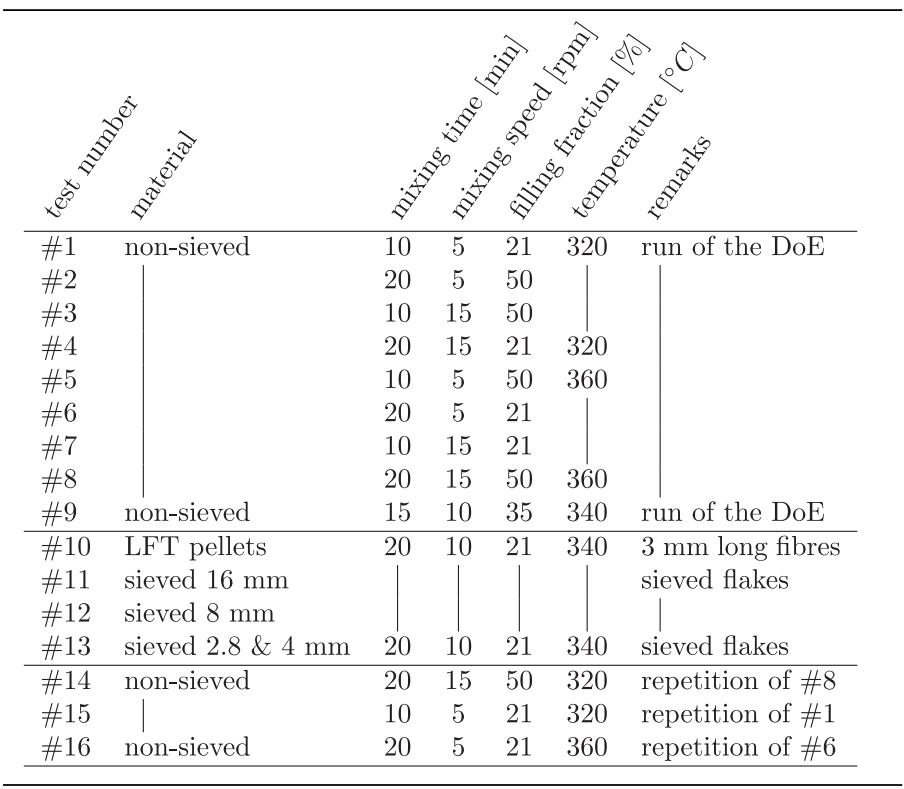

ing in a matter of seconds. For this study, the state of the material right after mixing was studied using cross-sectional microscopy. However, directly after mixing, the doughs are too porous to be prepared for microscopy. Therefore, the doughs were half-pressed to a thickness varying between $15 \mathrm{~mm}$ and $20 \mathrm{~mm}$. It was found that the compression of these doughs occurs in two stages. In the initial pressing stage, most void pockets close, while the dough does not flow outwards. During the second stage, the dough starts to flow and the remaining void pockets close. A dough thickness of about $15-20 \mathrm{~mm}$ was found to be the end of the first pressing stage. Note, in a separate study it was found that, after compression moulding of a dough, the porosity fraction is null or negligible, except in specific locations such as large thickness increases in the flow direction. [35]

It is assumed that the structure and arrangement of the fibres are identical in the extruded doughs and the half-pressed doughs, except for the out-of-plane orientation of fibres. Especially, it is assumed here that the local relative variations in fibre volume fraction and the clusters of fibres remain the same as in the non-pressed doughs.

The stochastic nature of the dough makes quantitative analyses of its microstructure by micrography difficult. However, an extensive microscopy study can provide sufficient data to obtain reliable statistical measurements. In the present study, the following procedure was adopted to gather data for measurements of the QoM. First, each half- pressed dough was cut to produce sections of roughly $10 \mathrm{~mm}$ width (see Fig. 6). Six sections were cut per dough - two from the front, the middle and the rear areas - and were then embedded and polished. Two large cross-sectional micrographs were taken for each section at a $\times 700$ magnification and were automatically stitched to $20,000 \times 20,000$ pixels each, representing an area of $6 \mathrm{~mm} \times 6 \mathrm{~mm}$. Hence, twelve large microscopic images were obtained per dough, representing an analysed area of roughly $430 \mathrm{~mm}^{2}$. Parts of such micrographs are shown in Fig. 7(a) and 7(e). Microscopic images of the non-mixed material, i.e. the flakes and LFT pellets, were also captured for the calculation of the IoS (see following section). Microscopic photographs of the other two materials, the injection moulded short fibre composites and the compression moulded flakes (see Section 3.1), were also made to compare their QoM to those of the doughs. Samples were water cut using a diamond coated saw and dried at $90^{\circ} \mathrm{C}$ for $12 \mathrm{~h}$. before they were embedded using an epoxy resin (EpoFix, Struers) and polished using a sequence of 600-1200-2000-4000 sanding papers and finaly a silica oxide suspension. A Keyence VHX digital microscope was used in all occasions, stitching was performed using the built-in automatic stitching function.

Image segmentation was executed automatically on each picture using Matlab software, using the peak locations of each image histogram to determine the fibre, matrix and porosity fraction. Details on this operation can be found in Appendix Appendix B. 


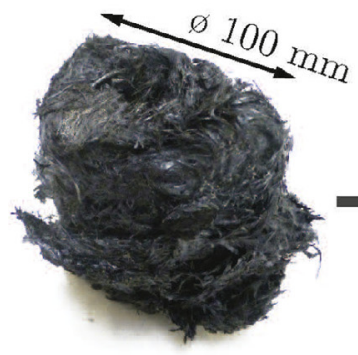

dough after mixing locations of the microscopy specimens

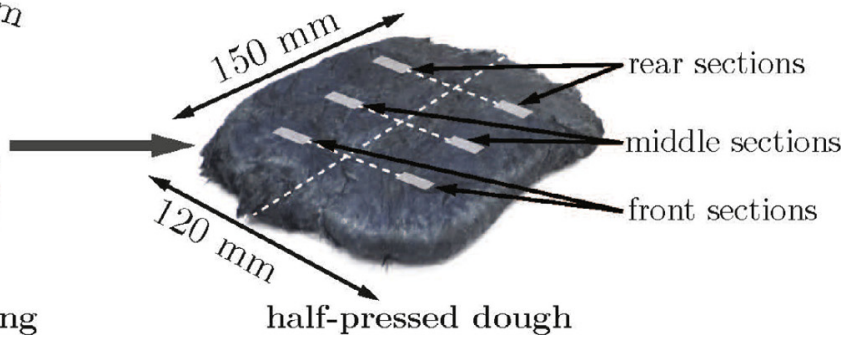

Fig. 6. Photographs of a dough after mixing and after being half-pressed. The locations of the microscopy specimens in the dough are highlighted in white on the half-pressed dough.

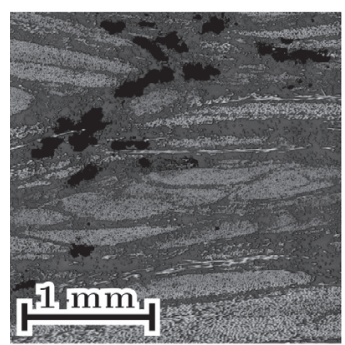

(a) initial image

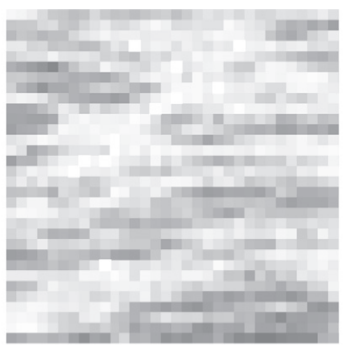

(b) $75 \mu \mathrm{m}$ grid CoV $=0.54$

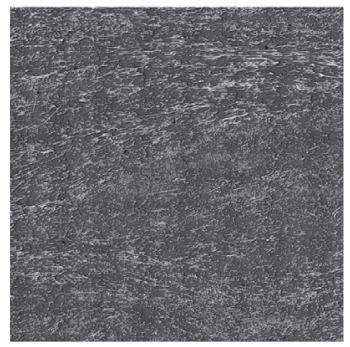

(e) initial image

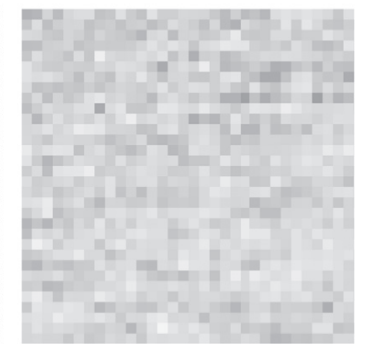

(f) $75 \mu \mathrm{m}$ grid CoV $=0.25$

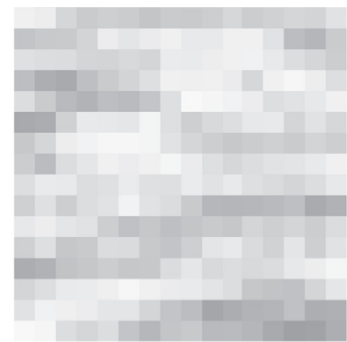

(c) $150 \mu \mathrm{m}$ grid CoV $=0.41$

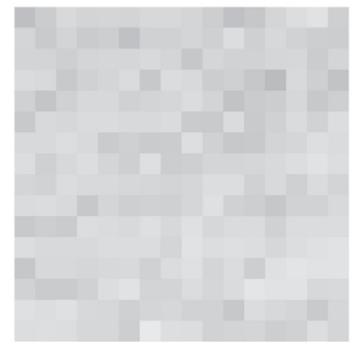

(g) $150 \mu m$ grid

CoV $=0.16$ (d) $300 \mu \mathrm{m}$ grid

CoV $=0.28$
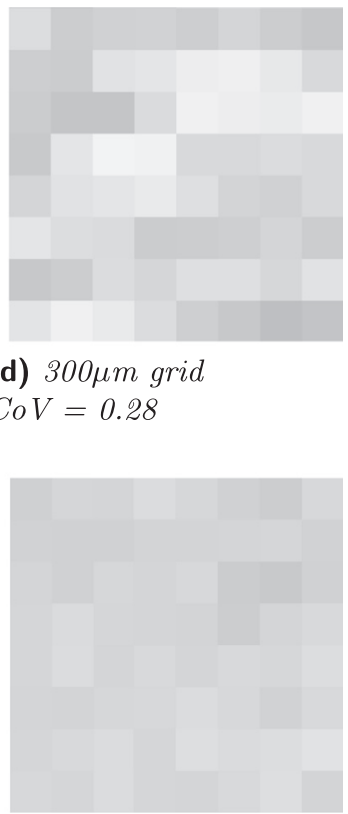

(h) $300 \mu m$ grid

CoV $=0.09$

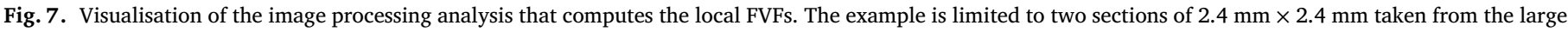

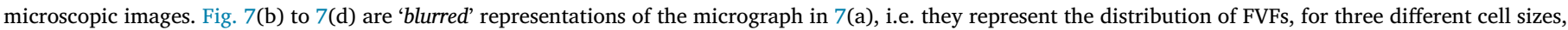

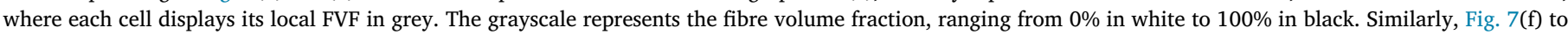
$7(\mathrm{~h})$ represent the FVF in the microscopic image in $7(\mathrm{e})$ for various cell sizes.

At first, a large-scale measurement of the evenness of the doughs was determined. For each dough, the FVFs of the twelve microscopic images were first calculated and then grouped by their location in the dough: front, middle or rear (see Fig. 6). This resulted in an estimate of the global variations in FVF, which will be analysed in Section 4.

\subsubsection{Intensity and scale of segregation}

The segmented cross-sectional micrographs are divided into a grid of various cell sizes (from $18.75 \mu \mathrm{m}$ to $600 \mu \mathrm{m}$ ). The FVF of each cell is computed, excluding the fraction of porosity, i.e. FVF $=$ Area $_{\text {fibres }} /\left(\right.$ Area $_{\text {totalcell }}-$ Area $\left._{\text {porosity }}\right)$. Note that if the porosity fraction in any cell is larger than $30 \%$, this cell is discarded from the analysis. The CoV of the local FVFs is recorded per image for each grid size. The same calculation was performed for the initially non-mixed materials, i.e. blends of flakes and polymer or pellets and polymer. The IoS is defined as the ratio of the $\mathrm{CoV}$ between two microscopic pictures: the half-pressed doughs over the initially non-mixed flakes - polymer combination (see Fig. 8 for an illustration of the method). Fig. 7(a) and 7(e) display two micrographs with a clear difference in QoM. The top picture presents large clusters of fibres with moderate evenness. The bottom picture shows the opposite: small clusters and considerable evenness.
Fig. 7(b) to 7(f) and 7(h)shows the grids of FVFs for various cell sizes (75 $\mu \mathrm{m}, 150 \mu \mathrm{m}$ and $300 \mu \mathrm{m}$ ) where the greyscale represents the FVF, ranging from $0 \%$ in white to $100 \%$ in black. It is clear from the grids that the CoV of the local FVFs will give different results for both micrographs, thus resulting in different degrees of IoS.

The calculation of SoS starts with the grid of local fibre fraction with cells of $18.75 \mu \mathrm{m}$, computed from the microscopy images. Image segmentation is performed on the grid into two phases with the FVF respectively below and above the median FVF (see Fig. 9). Considering the newly segmented image as a matrix, the maximum striation thickness of the high FVF clusters, i.e. the maximum height of the white regions in Fig. 9(e), is measured for each column. The average of the maximum striation thicknesses of all columns is calculated, which gives the measure of the SoS. Fig. 9 shows the grid of FVFs from the two micrographs in Fig. 7(a) and $7(\mathrm{e})$, as well as the segmentation by FVF. Fig. 9(b) has much larger clusters than Fig. 9(d), which are detected by the measure of the SoS, $400 \mu \mathrm{m}$ and $175 \mu \mathrm{m}$ respectively. This method is sensitive to the orientation of the micrographs in the cross-sectional specimen. However, all micrographs were taken with the same orientation to prevent this issue.

In addition, an artificial random distribution of fibres was created to represent the QoM of an almost perfectly even dough. For that, a 2D 
compute the $\mathrm{CoV}$ of

the local fibre fraction:

after mixing:
doughs

varying cell size: $18.75 \mu \mathrm{m}$ to $600 \mu \mathrm{m}$

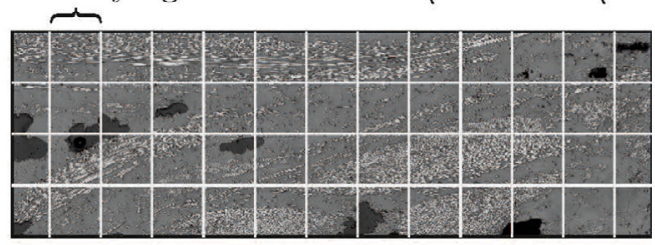

$\mathrm{FVF}_{\text {dough }}=20 \%$

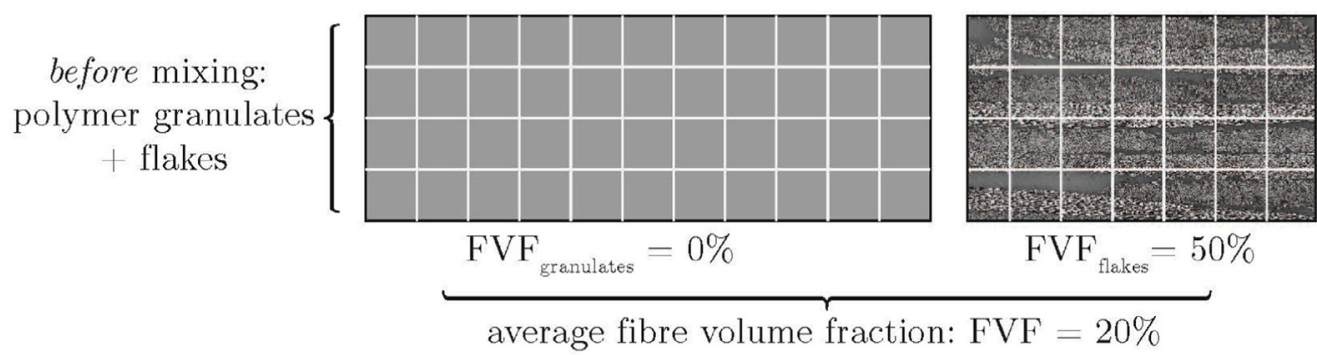

$$
\mathrm{IoS}=\frac{\mathrm{CoV} \text { of local fibre fraction (after mixing) }}{\mathrm{CoV} \text { of local fibre fraction (before mixing) }}
$$

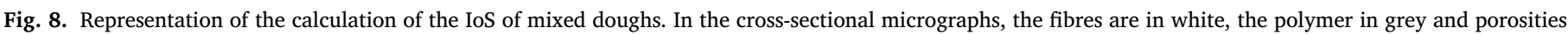
are in black. The same procedure is applied for the dough made of LFT pellets, for which the CoV before mixing is calculated from microscopic images of the initial LFT pellets.

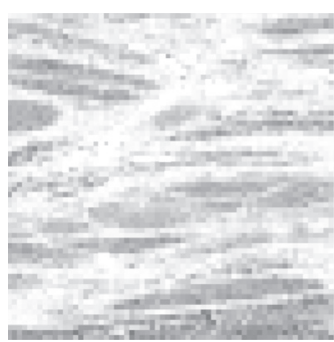

(a) $37.5 \mu \mathrm{m}$ grid

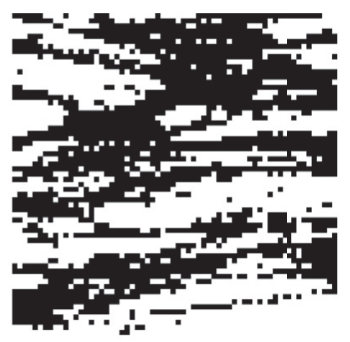

(b) $S o S=305 \mu \mathrm{m}$

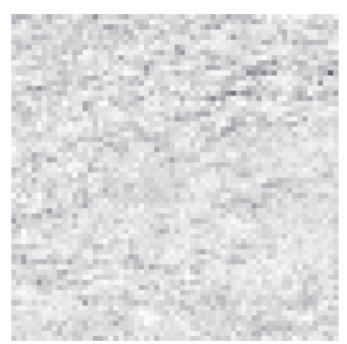

(c) $37.5 \mu \mathrm{m}$ grid

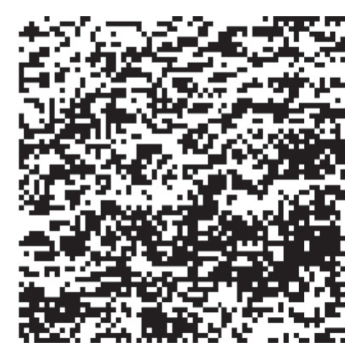

(d) $S o S=175 \mu \mathrm{m}$
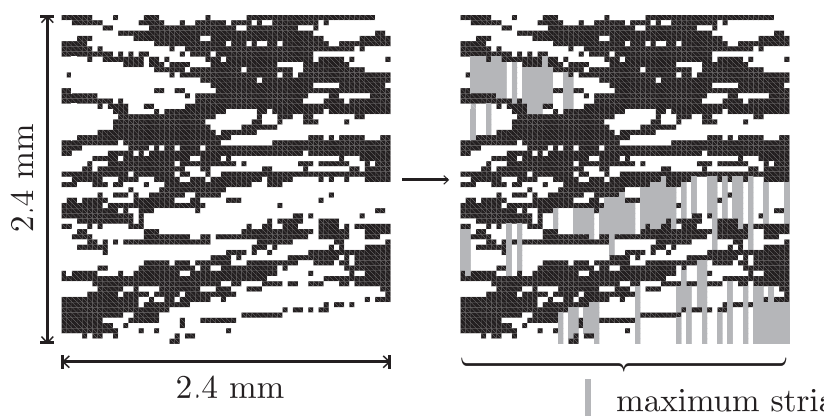

SoS average of the maximum striation thicknesses

this example $305 \mu \mathrm{m}$

(e)

Fig. 9. Fig. 9 (a) and 9 (c) show the local FVFs of the microscopic images of Fig. 7. Fig. 9(b) and 9(d) are the segmented images of 9 (a) and 9 (c) respectively, where the areas of higher fibre fraction are shown in white. The SoS is the average ofe striation thicknesses of the white regions. The bottom figure, (e), illustrates the calculation of SoS from the segmented images.

space was randomly filled with non-overlapping ellipses at $20 \%$ coverage, from which the IoS and SoS were measured. This random fill represents a mixing situation that is difficult to achieve in reality, but it is still far more segregated than a perfectly uniform distribution of fibres. This artificial random distribution helps to visualise the theoretical limit of evenness and clustering achievable for mixed C/PPS dough.

\subsubsection{Bundle size distribution}

An additional method was implemented to determine the size of the fibre clusters in each image, which can range from a single loose fibre to 3000 clustered fibres.

Images of the segmented fibres were used for this purpose. In the segmented micrographs presented here, adjacent fibres may appear to 


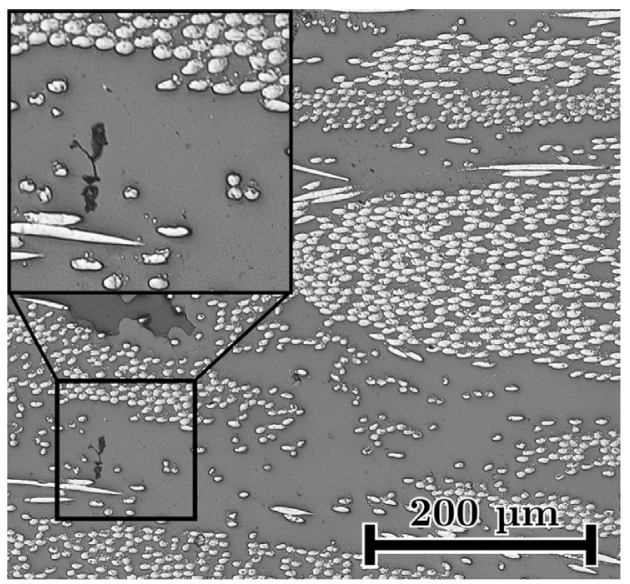

(a)

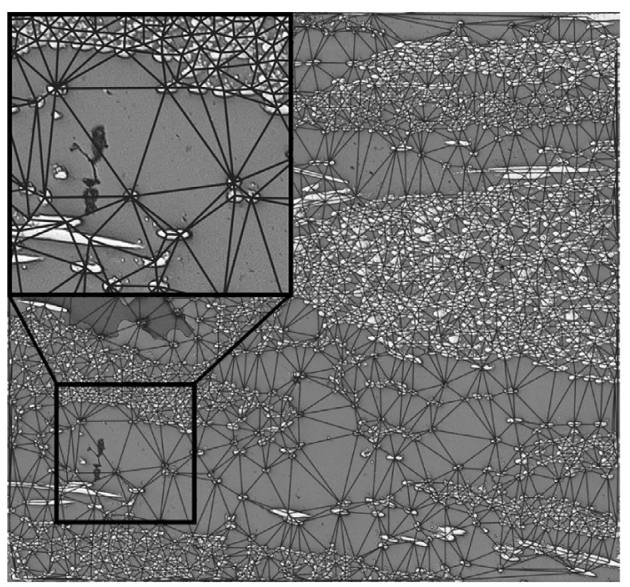

(c)

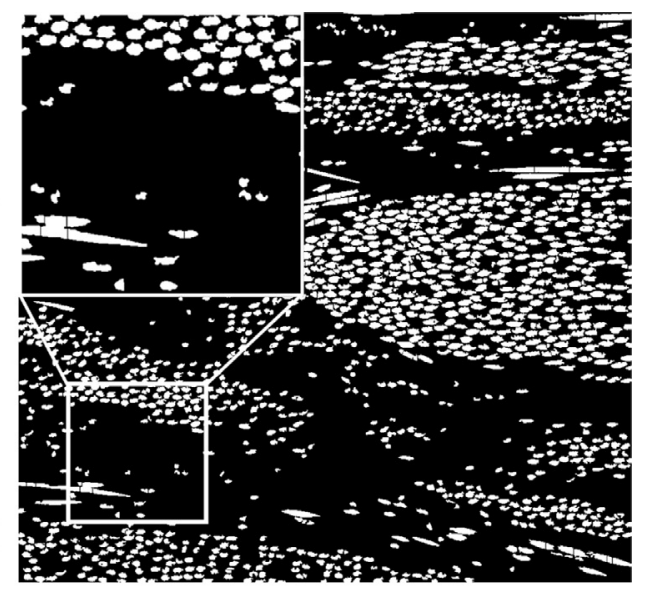

(b)

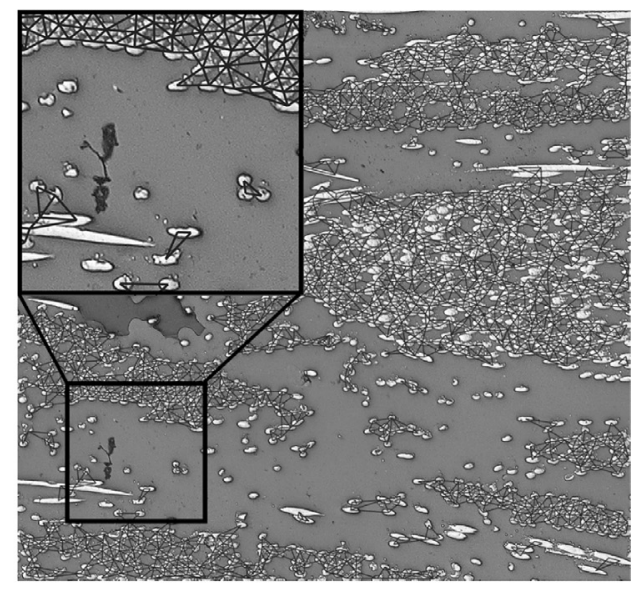

(d)
Fig. 10. Visualisation of the intermediate steps of the algorithm implemented to determine the BSD of cross-sectional micrographs. Fig. 10(a) shows the input cross-sectional micrograph while 10(b) displays the segmented fibres after the fibres were disjointed using a watershed. Fig. 10(c) and 10(d) show the Delaunay triangulation before and after the refinement technique (Section 3.3.2). The refined triangulation was used to compute the BSD in each micrograph. be joint because of the segmentation step. However, an accurate description of the clusters of fibres requires fully disjoint fibres. A watershed algorithm was applied to the segmented images to overcome this issue. Fig. 10(b) shows the segmented image after applying a watershed analysis. Most fibres are disjointed from one another. However, flat ellipses, which correspond to fibres parallel to the microscopic plane, may be cut by the watershed algorithm, thereby outputting multiple domains of a single fibre. These numerical artefacts seemed limited compared to the benefits of applying a watershed algorithm.

A Delaunay triangulation was then performed on all fibre centres on each image (Fig. 10(c)). A refinement technique was needed to cluster the fibres that are close to each other. Fig. 10(d) illustrates the result of the refinement technique. The clusters of fibres are clearly disjointed from one another, although some fibres are wrongly attributed to another cluster. This issue artificially increases or reduces the number of fibres per cluster, but the overall effect was found to be limited. The number of fibres per cluster is calculated from the list of connected components in the refined Delaunay graph, which is then converted to the bundle size distribution (BSD).

In the aforementioned refinement technique, all segments longer than three fibre diameters, corresponding to a regular hexagonal configuration at $10 \% \mathrm{FVF}$, are discarded. A corrected fibre-to-fibre distance was calculated to take into account ellipse eccentricities and orientations. A detailed description of the calculations can be found in Appendix Appendix C.
To provide additional overview on the various steps performed to obtain the BSD, IoS and SoS (previous section) from the microscopy images, a schematic flow chart is included in Appendix Appendix D.

\section{Results}

The half-pressed doughs listed in Table 1 were prepared for microscopy and analysed according to the methods presented in Section 3. Initially, the DoE was analysed to determine the effect of mixing settings on QoM. The results of the reproducibility study are presented next. Then, the effects of the various FLDs on QoM are analysed and discussed.

\subsection{Design of experiments}

Fig. 11 (a) shows the IoS for all cell sizes, of the distinct twelve micrographs made from dough \#5. The IoS is always smaller than one, which indicates a mixed state that is, as expected, less segregated than the initial non-mixed material. Besides, the IoS of the randomly filled 2D space is plotted under random fill with a dotted line (Fig. 11). It is most probable that the IoS of all doughs are bounded by one and the IoS of random fill. A hatched region was added to the figure to represent a domain of the IoS that cannot be reached for the doughs in this study. One may note the large scatter between these twelve lines, which is why a grey bounding region depicting the maximum and minimum values of 


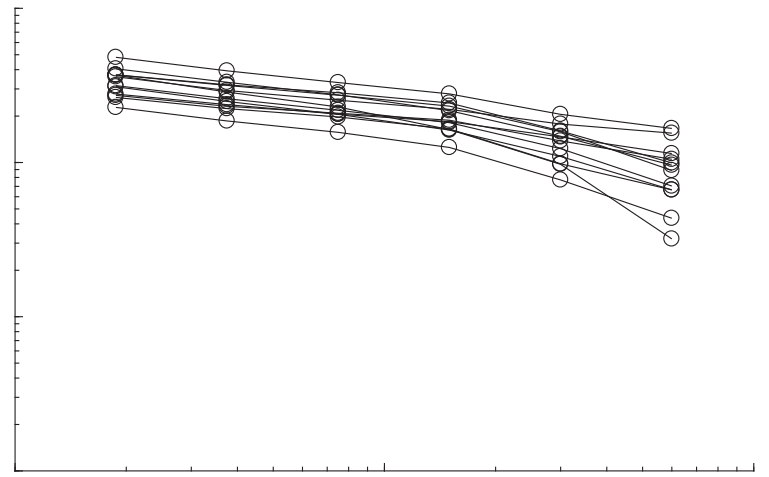

(a) IoS of the dough \#5

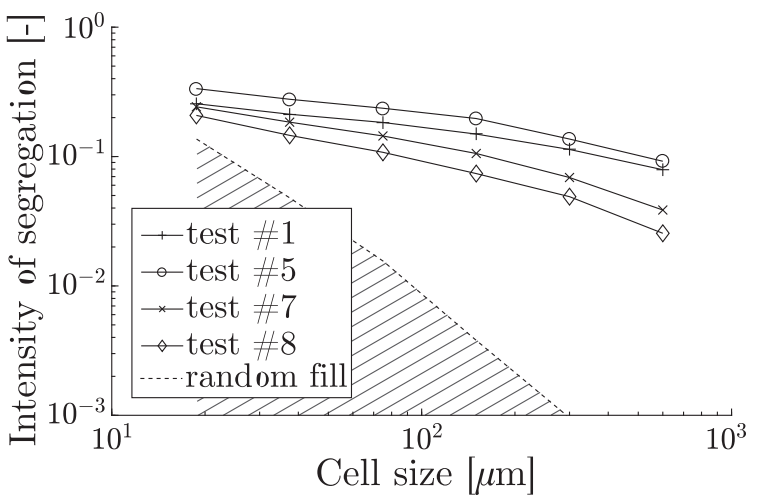

(c) Mean IoS of the doughs \#1, \#5, \#7 and \#8

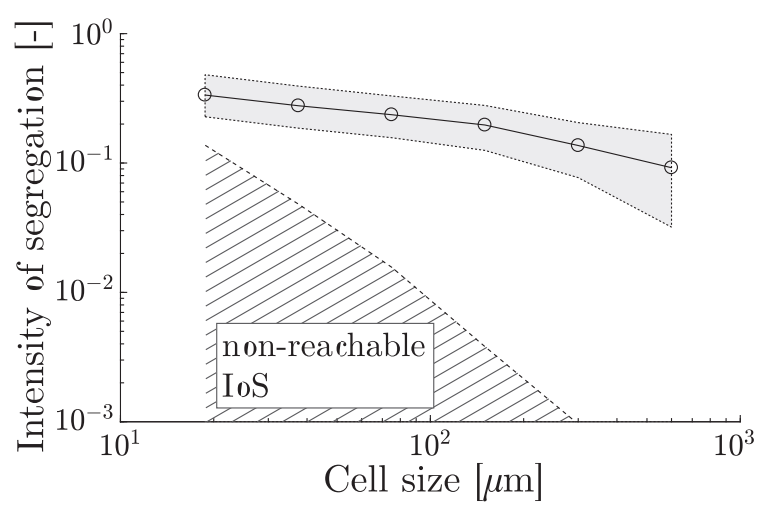

(b) IoS of the dough \#5

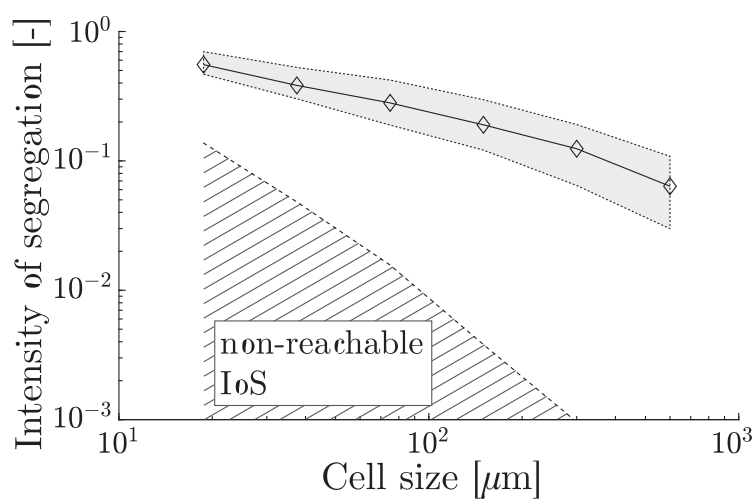

(d) IoS of the dough \#7

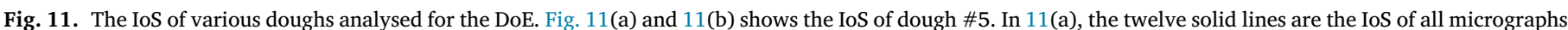

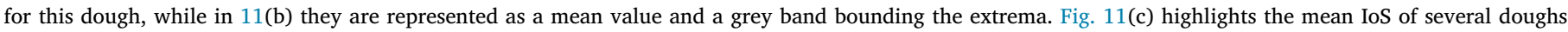
processed with various mixing settings. Fig. 11(d) shows the IoS of another dough with its mean value and extrema highlighting the variations of IoS in a dough.

the IoS are shown around the mean values plotted with a line as shown in Fig. 11(b). This graph illustrates that three measures are important:

- the mean IoS: it represents the evenness of the local FVF, as defined in Section 3.3.1. In order to make the analysis simpler, only the IoS for a cell size of $150 \mu \mathrm{m}$ is considered in detail for the DoE. This value was chosen due to its similarity to the initial bundle size, approximately $150 \mu \mathrm{m}$ in thickness at normal FVF. This size is assumed to be a relevant scale to determine the evenness of this material. Lower IoS values equal a better evenness of the fibres in the sample.

- The bandwidth of the IoS (height of the grey band), which corresponds to the intra-sample variability of evenness. The analysis of the DoE was performed only for the bandwidth at $150 \mu \mathrm{m}$, similarly to the mean of IoS. A small bandwidth indicates limited variability of evenness between the twelve micrographs.

- The effect of cell size on the IoS is described by the slope of the IoS as a function of cell size. It expresses the rate of change of evenness within the studied scale range. Steep slopes indicate that fibre bundles become evenly spaced at a large scale. Gradual slopes show that the local variations in FVF hardly decrease when increasing the scale.

An analysis of variance of the three measures of the DoE was performed. For all cases, a level of significance of 0.05 was selected, as it is common practice, to distinguish the effects that significantly influence the measures of the QoM.

The effects of the mixing settings on the mean IoS, the bandwidth and the slope are illustrated in Fig. 11. The mean IoS of several doughs is displayed in Fig. 11(c) where doughs \#1 and \#5 were processed at a low mixing speed and for a short amount of time, whereas doughs \#8 and \#7 were processed at a high mixing speed and for varying amounts of time.

A first consideration showed that increasing the mixing speed decreased the bandwidth. Both the mixing speed and mixing time were found to decrease the mean IoS. The mixing speed, mixing temperature and mixing time were all found to decrease the slope of the mean IoS.

Further, Fig. 11(a) shows the intra-sample variability, which can be of a similar magnitude as the variations between the IoS of doughs made with various settings (Fig. 11(c)). This urged us to reconsider the reliability of considering the mean IoS and bandwidth separately. Therefore, the analysis of the DoE was carried out for six repetitions, corresponding to the six sections in each dough. The results show again that mixing speed and mixing time influence the IoS; with a more significant effect (p-value 5 to 10 times lower) as compared to the analysis of the mean IoS. The strength of the significant factors is similar for both studies, with mixing speed having stronger influence than mixing time. As a conclusion, although intra-sample variability is high, the mixing speed and mixing time have a strong and significant effect on the IoS. Furthermore, the analysis of variance showed that the effects of the filling 

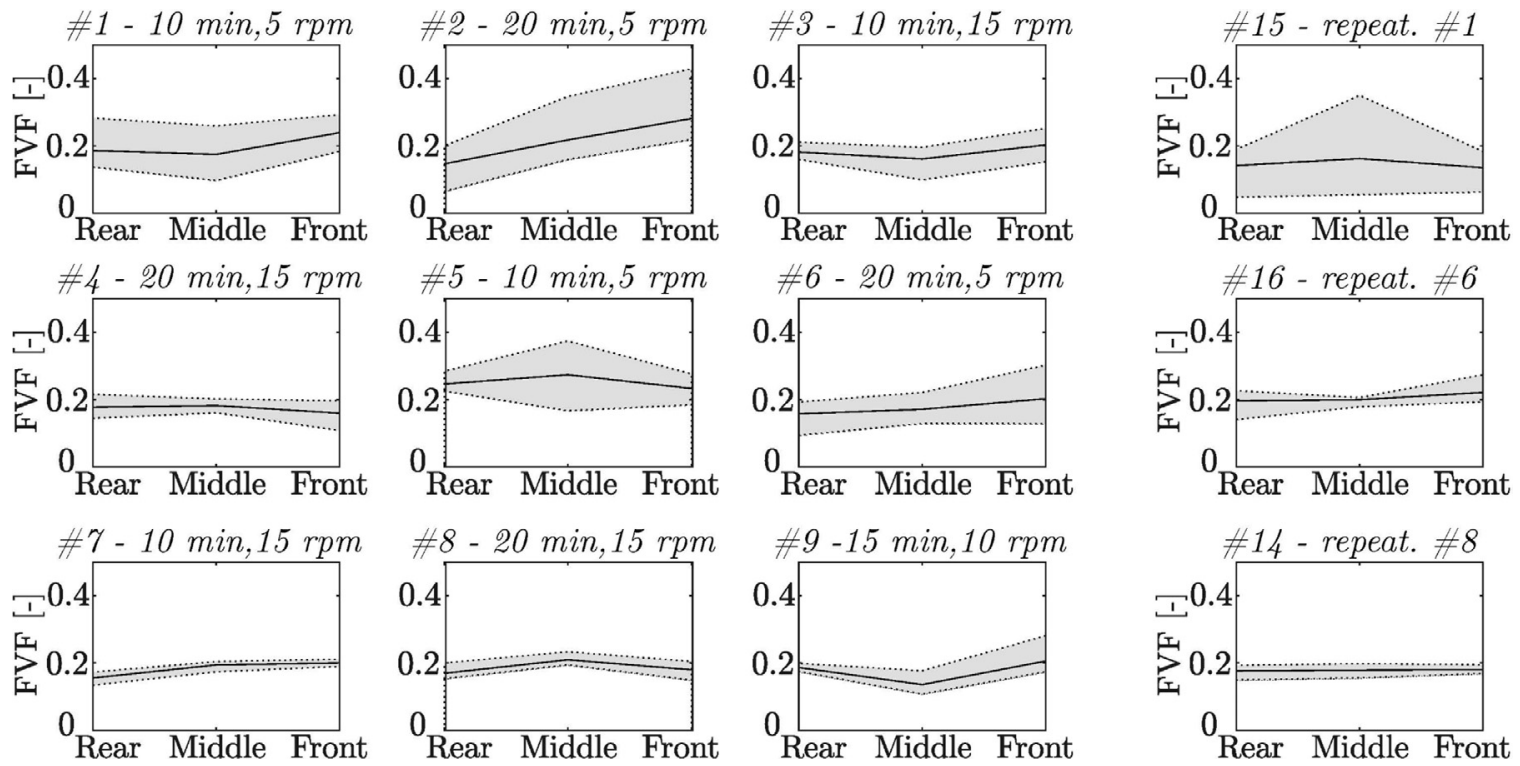

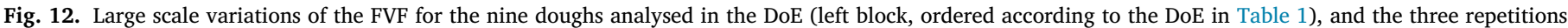
(right block) for the reproducibility study. The black lines and the grey bands show the mean and the extrema respectively.

fraction and mixing temperature are not significant. As a consequence, their effect is not detailed. The effect of interactions between multiple parameters could not be determined with the DoE carried out (resolution IV).

Next, the SoSs of the doughs were analysed. The average SoS of the nine doughs was found to vary between $260 \mu \mathrm{m}$ and $420 \mu \mathrm{m}$ (see Table A. 3 in the Appendix), which is a small range, when compared to the SoS of the random fill $(101 \mu \mathrm{m})$ and indicates that fibre-dense regions are of comparable size. However, the intra-sample variation of SoS varies considerably, from $150 \mu \mathrm{m}$ to $430 \mu \mathrm{m}$. Again, mixing time and mixing speed were found to reduce the SoS.

The study of the effect of the mixing settings on the IoS and SoS showed that both the total amount of shear (mixing time and mixing speed) and the shear rate (mixing speed) significantly improve QoM on multiple levels: they reduce the IoS, SoS and the intra-sample variability. However, the low-shear mixer does have some limitations. A comparison of the IoS and SoS obtained for the best mixed doughs with those of the random fill confirms that the evenness and clustering of the doughs can still be improved, at least from a theoretical point of view.

Studying the microscopic images also revealed that fibres are far from evenly distributed or aggregated in small clusters, even after long mixing times and high mixing speeds, although it is also clear that longer mixing time and higher speed improve evenness and clustering. This phenomenon is highlighted in Fig. 12 which also shows the variations of FVF at a large scale, where the FVF of each micrograph is calculated and grouped according to its dough and its location in this dough. The solid line and the grey band represent the mean and extrema of the FVFs for each location respectively. The doughs processed at $5 \mathrm{rpm}$ for $10 \mathrm{~min}$ and $20 \mathrm{~min}-\# 1 \& \# 5$, and $\# 2$ \& \#6 respectively - all show very large variations in FVF, whereas FVF variations are much lower for the doughs processed at $15 \mathrm{rpm}(\# 3, \# 4, \# 7$ and \#8). This seems to indicate that mixing speed has a more pronounced effect than mixing time. The results of the analysis of variance also confirmed this for the IoS and the SoS. Additionally, the micrographs show that a combination of large and small fibre clusters are found in doughs mixed at $5 \mathrm{rpm}$. Fewer large clusters are found in dough processed at $15 \mathrm{rpm}$. This result is visualised in Fig. 13(b), which plots the BSD of several doughs. This figure is the output of the refined Delaunay triangulation and shows the volume fraction of bundles of every given size. The fractions of large bundles $(\geqslant 1,000$ fibres) and small bundles ( $\leqslant 100$ fibres) indicate whether the initial tows of 3,000 fibres were well dispersed. The figure shows that doughs \#1 and \#6 (5 rpm) had very similar BSDs with more than $30 \%$ of their volume consisting of large bundles, while the BSD of \#8 (20 min, $15 \mathrm{rpm}$ ) results in a much lower fraction of large bundles.

In conclusion, the analysis of variance shows that mixing time and speed improve all measures of QoM, with mixing speed having a stronger effect than mixing time. This observation was confirmed by analysing the BSD, the large-scale variations of FVF and the micrographs of doughs.

\subsection{Reproducibility}

The previous results showed that intra-sample variability is of great importance, and the same might apply to inter-sample variability. Three doughs made with various mixing settings (\#1, \#6 and \#8) were replicated (\#15, \#16 and \#14, respectively) to investigate the reproducibility of the mixing process. The mean IoS of the six doughs is plotted in Fig. 13(a). Their large-scale variations in FVF are displayed in Fig. 12. The findings from the previous section indicate that dough \#1 has a moderate QoM for all measures. Dough \#6 showed some improvement due to a longer mixing time. Dough \#8 demonstrates that it is among the doughs with the highest QoM in the study due to a long mixing time and a high mixing speed.

Limited inter-sample variability would lead to a clear grouping of IoS by mixing settings: \#1 \& \#15, \#6 \& \#16, and \#8 \& \#14. However, the measurements in Fig. 13(a) show non-groupable lines for all doughs. The situation is different, however, when looking at the large-scale variation of FVF (Fig. 12). Clearly, doughs \#1 and \#15 have very high variations of FVF. Doughs \#6 \& \#16 show reduced variability, whereas \#8 \& \#14 exhibit very limited variations of FVF. Additionally, the BSD was also analysed for these six doughs (Fig. 13(b)). Two separate groups can easily be identified. The BSDs of doughs \# 8 \& \#14 are clearly distinct from the other four: they have a higher fraction of small bundles (smaller than sixteen fibres) and a small fraction of large bundles (larger than 1000 fibres). The other four doughs cannot, however, be grouped by mixing settings, as was the case for the mean IoS. The fraction of large bundles in these doughs is nonetheless considerable, ranging from $20 \%$ to $40 \%$. Dough \#16 is particularly striking, as it has the second worst IoS and 


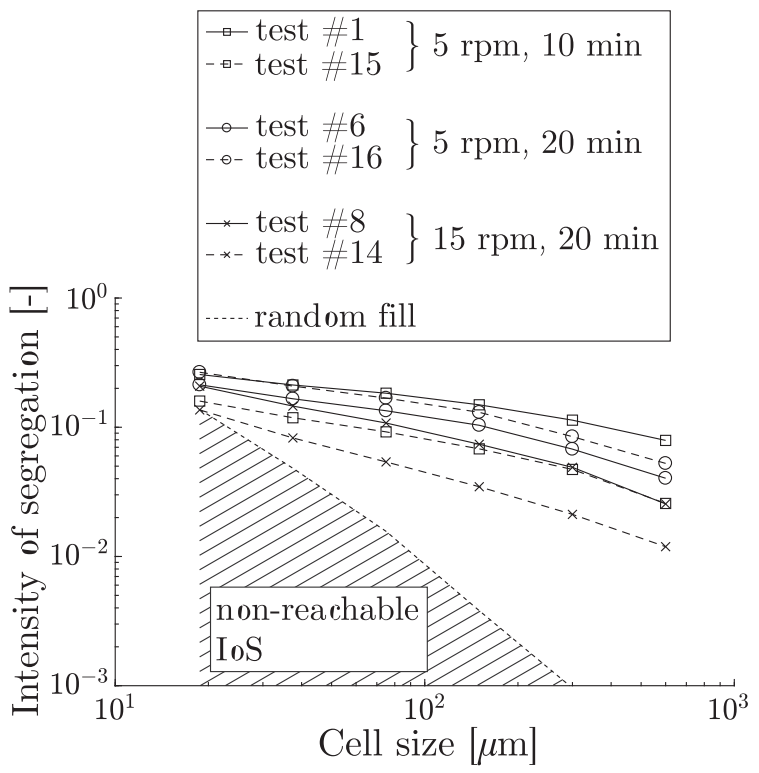

(a)

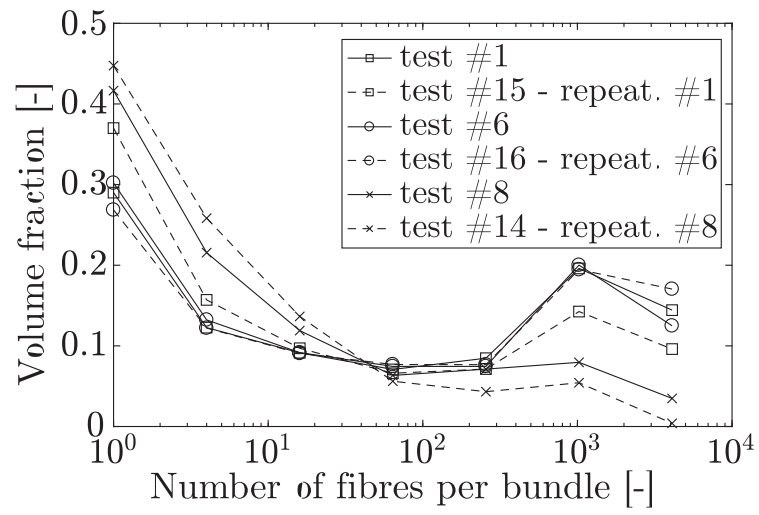

(b)

Fig. 13. The IoS and the BSD of the doughs analysed for the reproducibility study.

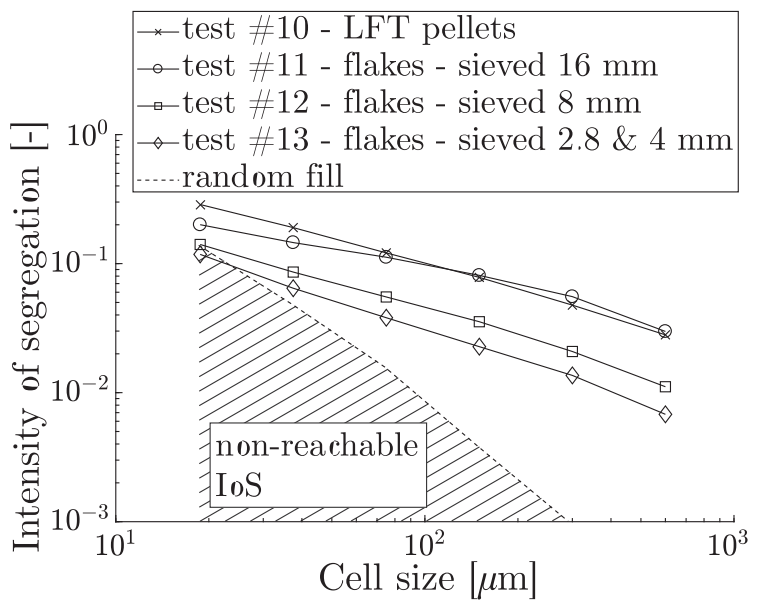

(a)

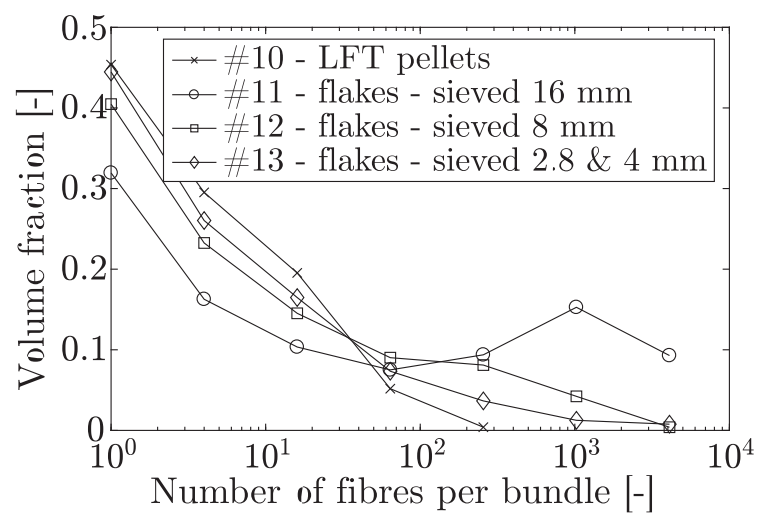

(b)
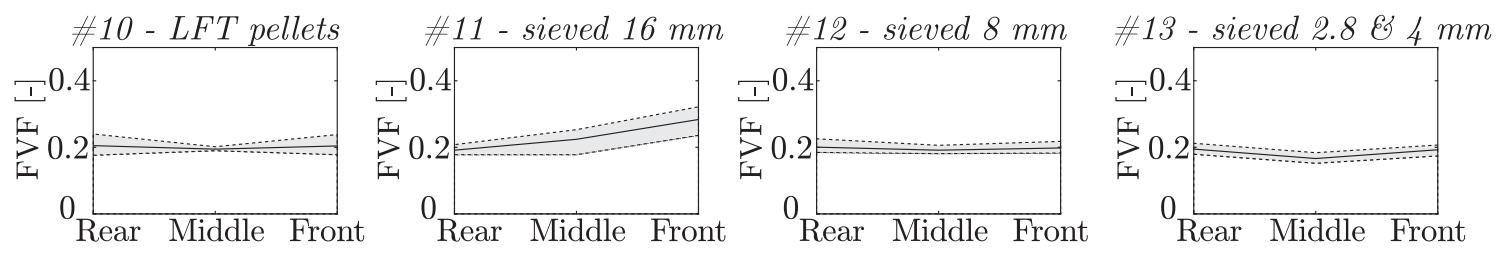

(c)

Fig. 14. (a) The IoS, (b) the BSD and (c) the large scale variations of FVF of the doughs processed with various FLDs.

the largest fraction of large bundles, but a small large-scale variation of FVF (Fig. 12). Nevertheless, its SoS and the slope of the mean IoS are average (see Table A.3 in the Appendix section).

\subsection{Effect of FLDs}

The last set of experiments concerns the effect of the FLD on the QoM. Sieved flakes and LFT pellets were used as described in Section 3. Fig. 14 aggregates the IoS, the BSD and the variations of FVF for the four doughs \#10, \#11, \#12 and \#13. Table 2 summarises the measures of the QoM for the four doughs. It also shows the standard deviation of the local FVF for the two comparison materials and the randomly filled 2D space. As a reminder, the IoS is defined as the improvement in evenness of the local FVF due to mixing. The results show that the evenness of LFT pellets improves less than for the other three doughs. However, its standard deviation of FVF is lower than for the doughs, showing a more even distribution of fibres. The standard deviations in Table 2 also highlights the differences between the compression moulded flakes (non- 
Table 2

Summary of the measures of the QoM for the doughs processed with various FLDs. The standard deviations of local FVF are also listed for two comparison materials and the randomly filled space.

\begin{tabular}{|c|c|c|c|c|c|c|}
\hline dough & material & $\begin{array}{l}\text { mean SoS at } \\
18.75 \mu m[\mu m]\end{array}$ & $\begin{array}{l}\text { mean IoS at } \\
150 \mu \mathrm{m}\left[\times 10^{-1}\right]\end{array}$ & $\begin{array}{l}\text { bandwidth at } \\
150 \mu m\left[\times 10^{-1}\right]\end{array}$ & slope of the IoS [-] & $\begin{array}{l}\text { standard deviation } \\
\text { of the FVFs at } \\
150 \mu \mathrm{m}\left[\times 10^{-1}\right]\end{array}$ \\
\hline \#10 & LFT pellets & 177 & 2.0 & 0.64 & -0.67 & 0.30 \\
\hline \#11 & sieved $16 \mathrm{~mm}$ & 310 & 1.6 & 0.54 & -0.53 & 0.79 \\
\hline \#12 & sieved $8 \mathrm{~mm}$ & 224 & 0.94 & 0.35 & -0.72 & 0.52 \\
\hline \multirow[t]{4}{*}{ \#13 } & sieved $2.8 \& 4 \mathrm{~mm}$ & 207 & 0.67 & 0.13 & -0.80 & 0.42 \\
\hline & \multicolumn{5}{|c|}{ compression moulded non-mixed flakes } & 1.49 \\
\hline & \multicolumn{5}{|c|}{ injection moulded short fibre composites } & 0.35 \\
\hline & \multicolumn{5}{|c|}{ artificial random fill } & 0.17 \\
\hline
\end{tabular}

mixed), the moulded LFT and short fibre pellets (see Section 3.1), and the doughs made from sieved flakes: the evenness of the smallest flakes is almost as good as the moulded short and long fibre composites.

The effect of flake size and FLD on QoM is evident for all measurements. Shorter fibres reduce IoS and SoS, and lead to a steeper slope of the IoS. The intra-sample variations for IoS and SoS steadily decrease with the reduction in FLD (see Table 2). Additionally, in the dough processed with the smallest flakes, only $2 \%$ of bundles were larger than 1,000 fibres, which is far lower than the $20 \%-40 \%$ range obtained for large flakes (Figs. 14(b) and 13(b)). However, there is still a large difference between the BSDs of the dough made from LFT pellets and from the smallest flakes, but this was expected due to the completely different nature of the input material. In addition, the large-scale variations of FVF mainly reduce from large to medium-size flakes. The doughs \#10, \#12 and \#13 all show similar large-scale variations of FVF, for which no improvement is noticeable with a reduction in fibre length. This may be a limit for the prototype mixer used in this study (Fig. 14(c)).

\section{Discussion}

When considering the whole recycling route studied in this article, the QoM obtained during the mixing phase affects the flow behaviour of the material in the mould and its mechanical properties afterwards. The industrial application of this recycling technology requires that moulded components have consistent mechanical properties, and hence low intra-part variability and good reproducibility. Additionally, the flow behaviour of the dough is partially determined by the number of bundle-bundle interactions, which translates to the bundle aspect ratio among others $[36,37]$. The homogeneity and reproducibility of the flow behaviour of the doughs are also important factors, which can be improved by reducing intra- and inter-sample scatter. The conditions necessary to address these issues can be derived from different aspects of the QoM. This leads to several measures of QoM that are to be either minimised or be optimised.

- Limited large scale variations of FVF are required. Figs. 12 and 14(c) demonstrate that variations of just a few percent can be achieved.

- The IoS and the intra-sample variability of IoS are other properties to be minimised. A locally even distribution of FVF helps reduce variability both in terms of rheology and part performance.

- A high reproducibility of QoM, all aspects combined, is also a key to the successful application of such a technology.

- BSD has a major effect on both flow behaviour and mechanical performance. On one hand, a considerable fraction of small bundles drastically increases the number of fibre-fibre interactions, which increases the apparent viscosity [36], where the upper limit is set by the flow distance in the mould and the size of the intricate features to fill. On the other hand, a substantial fraction of large bundles reduces the average bundle aspect ratio, which tends to limit the theoretical mechanical properties of the moulded component [38].
Nonetheless, it is rather difficult to draw a conclusion on the optimum BSD without a proper study of its effect on the flow behaviour of doughs as well as on the mechanical properties of moulded parts.

Regarding the list of criteria above, well mixed doughs (long mixing time, high mixing speed) do show improvement of IoS, intra-sample variability of IoS, and large-scale variations of FVF. It was, however, found that the reproducibility of doughs is moderate for all measured aspects of QoM, although only two doughs were analysed per setting. This lack of reproducibility can also be caused by the limitations in sample size: twelve microscopic images of $6 \times 6 \mathrm{~mm}^{2}$ per dough. In terms of the process window, the oxidation of the polymer is more severe for long mixing times in the presence of oxygen, which should be prevented in order to limit the degradation of the polymer. The results also pointed out that increasing the mixing speed is a more effective way to improve QoM than increasing mixing time. Thus, the authors recommend increasing the mixing speed to limit polymer degradation, although high shear rates may break fibres during mixing. This has not been a problem yet with the current mixing prototype, which has technical limitations that prevent its use at higher rpm. In addition, it was found that the SoS of all the doughs were close, and of the same order of magnitude; in comparison to other finely-clustered materials, i.e. the injection-moulded short-fibre composites and the artificial random distribution of fibres. On the contrary, the BSD varied drastically with respect to mixing settings and FLDs. Hence, it is supposed that BSD and SoS do not exactly characterise the same aspects of QoM, or do not characterise them with the same strength. BSD is an important material property, as explained in the previous bullet list. This may indicate that the method chosen to measure SoS is not the most suitable for this type of material or mixing technology.

Concerning the effect of FLD on the QoM of doughs, large improvements are noticed between doughs \#11/sieved $16 \mathrm{~mm}$ and \#12/sieved 8 $\mathrm{mm}$ for all measures of QoM. These improvements seem to saturate between \#12/sieved $8 \mathrm{~mm}$ and \#13/sieved $2.8 \& 4 \mathrm{~mm}$; and the large-scale variations of FVF, in particular, are comparable (Fig. 14(c)). Therefore, the authors recommend using small flakes in this process, especially with a mean fibre length smaller than $15 \mathrm{~mm}$.

\section{Conclusions}

An experimental study was carried out to understand how to improve the QoM of mixed doughs, made of long-fibre-reinforced polymers. Contrary to other LFT processes and materials, the flake material and the mixer used in this study are unconventional. The material consisted of multi-layered, woven-fibre reinforced composites plus polymer granulates. The mixer had a very low rotation speed to prevent fibre breakage, thereby limiting blending efficiency. Several aspects of QoM were selected from literature, namely evenness and clustering, and implemented for this study. The experimental methods involved image analysis of a large set of cross-sectional micrographs. The analysis was based on quadrats to measure IoS and SoS, and on refined Delaunay triangulation to measure BSD. Choices were made to 
measure the following aspects from the extensive set of cross-sectional micrographs:

- IoS, or evenness of the local FVFs.

- SoS, representing a measure of the clustering of fibres.

- Intra-sample variations of IoS, SoS and FVFs.

- BSD, describing the size distribution of fibre clusters.

The methods developed and implemented in this paper were found adequate for the material in this article, as well as to characterise the QoM of other LFTs. The refinement technique performed on the Delaunay triangulation is definitely beneficial, as it quantifies a material property of LFTs: bundle sizes. This property can also be used for the micro-mechanical modelling of LFTs. The IoS is a good measure to compare other mixing devices to the one used in this study, or to compare the doughs with other materials. Further developments of this recycling technology or of LFT material should consider the measurement of the IoS as a benchmark, similar to the current practice for polymer blends [39]. Finally, it is not sure whether the current implementation of SoS measurement provides information beyond the IoS and BSD.

The effects of mixing time, mixing speed, temperature and filling ratio on QoM were analysed by performing a fractional factorial DoE. It was found that the mixing time and mixing speed are the main drivers to improve QoM for all measures. The reproducibility of the QoM in doughs was inspected and was found to be marginal. On the other hand, the effect of the FLDs on QoM was clear for all measures: smaller flakes significantly improve the QoM. The recommendations for better mixing for this particular mixing setup are first to shred scrap to a smaller size, yielding a mean fibre length under $15 \mathrm{~mm}$. In addition, further improvements can be made to the mixing machine by blending at higher shear rates. This can be achieved by modifying the mixer design, but special care should be taken to minimise fibre breakage. It is still unclear whether the best QoM obtained in this study is sufficient. Further work is required to understand the effect of QoM on the flow behaviour of doughs and, subsequently, on mechanical properties of moulded parts.

\section{Declaration of Competing Interests}

The authors declare that they have no known competing financial interests or personal relationships that could have appeared to influence the work reported in this paper.

\section{Acknowledgments}

This project was financed by the Dutch Organisation of Applied Research SIA, through the project grant SIA-RAAK 2014-01-72PRO. The authors are grateful to the project partners: Toray Advanced Composites, GKN Fokker, Cato Composite Innovations, Dutch Thermoplastic Components and Nido RecyclingTechniek. The authors are thankful to Sotiris Koussios and Waqas Ali for internal review of the manuscript.

\section{Appendix A. List of the QoM measurements}

\section{Appendix B. Image segmentation and correction}

The image segmentation of all cross-sectional micrographs was automatically performed by the peak locations of the image histogram. A section of a micrograph is shown in Fig. B.15(a); its histogram is shown in Fig. B.15(b). The histogram contains a grey level peak for the epoxy (mounting resin for polishing), the PPS matrix and the fibres. The average grey level between epoxy \& matrix, and between matrix \& fibres is chosen to segment the images in the three phases (see the image segmentation axis in Fig. B.15(a)).

The magnified fibre at the bottom-left corner of Fig. B.15(a) emphasizes a black contour around the fibre, being an optical artefact captured by the microscope. Such a defect systematically lowers the measured FVF of the image. Therefore, a global correction was performed for the FVF of each image. It was assumed that a large number of micrographs was enough to represent the correct FVF of the samples, $20 \%$ on average. The global average FVF of the sixteen doughs $\times$ twelve micrographs was set to $20 \%$. The results of this correction are shown in Figs. 12 and 14(c).

\section{Appendix C. Centre-to-centre distance correction}

The micrographs studied in this article are cross-sections of discontinuous fibre composites. The cross-sections of cylindrical fibres with the micrography plane are ellipses with various eccentricities and orientations. In Section 3.3.2, the Delaunay triangulation of the ellipse centres is refined by discarding centre-to-centre segments longer than a threshold distance. This refinement technique is required to cluster the various fibre bundles. However, the Euclidean centre-to-centre distance in the plane of observation does not rigorously measure the distance between the fibres.

Consider the following situation; two ellipses in contact along their major axes in the microscopy plane have a longer Euclidean centre-tocentre distance than two touching fibres whose cross-sections are circles. (see Fig. C.16 (a)). Yet, the true distance between the centres is one fibre diameter in both cases. The relation with the observed distance depends on the orientations and eccentricities of the two ellipses. Thus, a corrected centre-to-centre distance is required to accurately distinguish the various clusters. The objective of this section is to define such a corrected distance.

The strategy implemented here is to consider a sleeve of polymer growing radially around two fibres until the sleeves touch. When the fibres are cut in the micrography plane, the cross-sections of the sleeves are also elliptical with the same orientation and eccentricity as their parent fibre (see Fig. C.16 (b) to (d)). In the micrography plane, a nonnormal orthogonal basis can be associated with each fibre and its sleeve (see Fig. C.17). The elliptical sleeves grow radially and isotropically in their respective bases.

At the junction point of the two sleeves (Fig. C.16 (b) to (d)), the sleeves have grown by the same amount in their respective bases. The corrected centre-to-centre distance, noted $d_{c}$, corresponds to the size of these sleeves of polymer and is in all cases $\leq d_{0}$. This distance is related to the change-of-basis matrices of each ellipse's basis and the direction of $\overrightarrow{P P^{\prime}}$, as elaborated mathematically in the following paragraphs.

Figure C.17 illustrates the cross-section of two fibres $F_{P}$ and $F_{P^{\prime}}$, in the plane of observation. The fibres are oriented differently from each other, leading to different ellipse orientations and eccentricities in the cross-sectional plane, whose centres are noted $P$ and $P^{\prime}$. Each ellipse is determined by its major axis, minor axis and orientation in the reference orthonormal basis of the cross-sectional plane, $\mathcal{B}_{0}$ : (a,b, $\left.\theta\right)$ and (a', $\left.\mathrm{b}^{\prime}, \theta^{\prime}\right)$ respectively for $F_{P}$ and $F_{P^{\prime}}$. For all fibres analysed in this paper, $b^{\prime}=$ $b=$ fibre diameter. Let $\vec{\Omega}$ be the unit vector in $\mathcal{B}_{0}$ collinear to $\overrightarrow{P P^{\prime}}$.

Additionally, consider an orthogonal basis for each ellipse, $\mathcal{B}$ and $\mathcal{B}^{\prime}$, whose unit vectors correspond to the axes of the ellipses. The changeof-basis matrix from $B$ to $\mathcal{B}_{0}$ is:

$\mathbf{T}=\left(\begin{array}{cc}\frac{a}{b} \cos (\theta) & \frac{a}{b} \sin (\theta) \\ -\sin (\theta) & \cos (\theta)\end{array}\right)$

Therefore, $\mathbf{T}^{-1} \vec{\Omega}$ is the unit vector $\vec{\Omega}$ expressed in $\mathcal{B}$, and $\left\|\overrightarrow{P P^{\prime}}\right\| \times$ $\left\|\mathbf{T}^{-1} \vec{\Omega}\right\|$ represents the length $\left\|\overrightarrow{P P^{\prime}}\right\|$ expressed in basis $\mathcal{B}$. Similarly, $\left\|\overrightarrow{P P^{\prime}}\right\| \times\left\|\mathbf{T}^{\prime-1} \vec{\Omega}\right\|$ is computed for the basis $\mathcal{B}^{\prime}$.

As stated above, the elliptical sleeves grow radially and isotropically in their respective bases. If one of the ellipses were a circle, the sleeve 
Table A1

Summary of the measurement results of the QoM for the samples studied in this article.

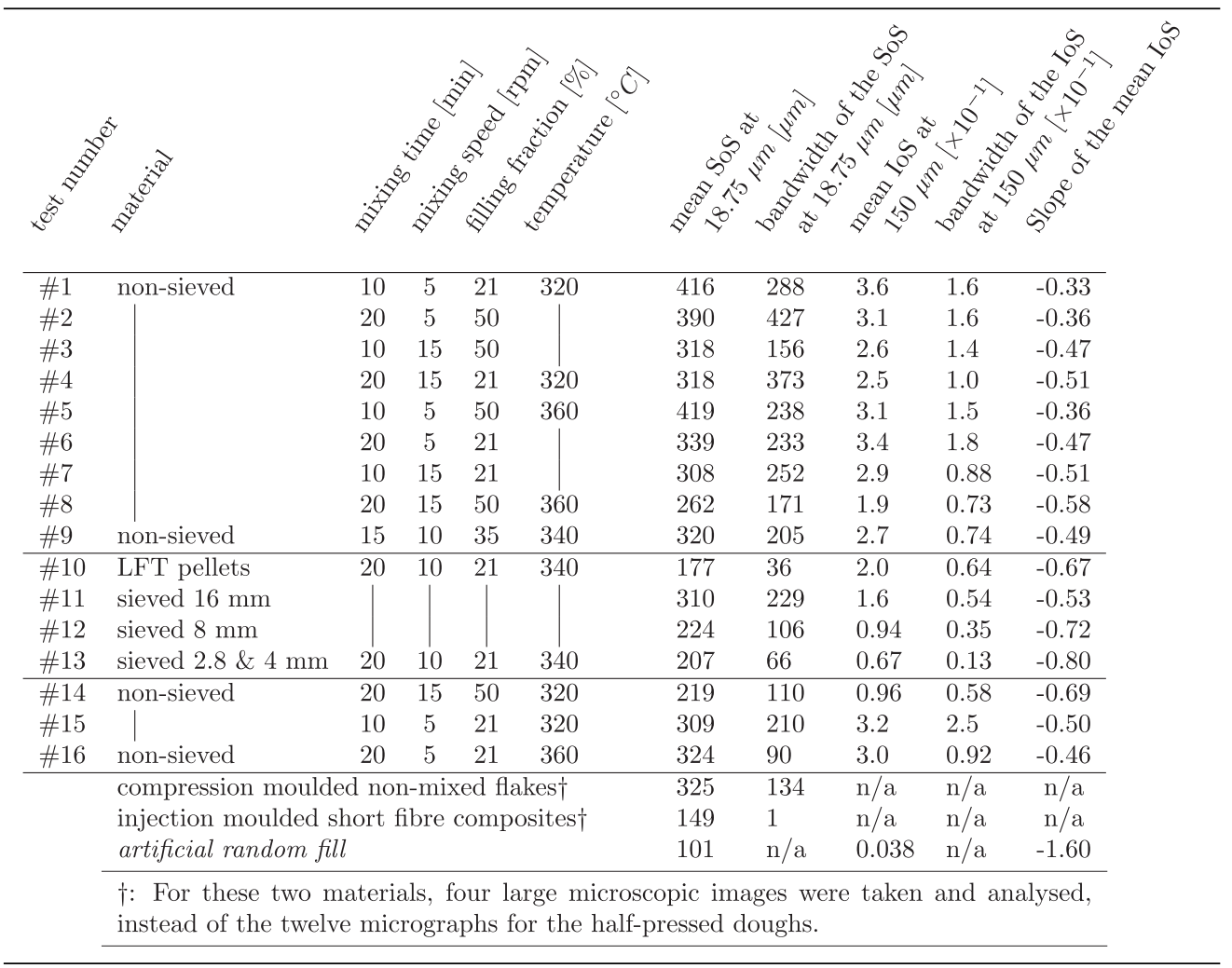

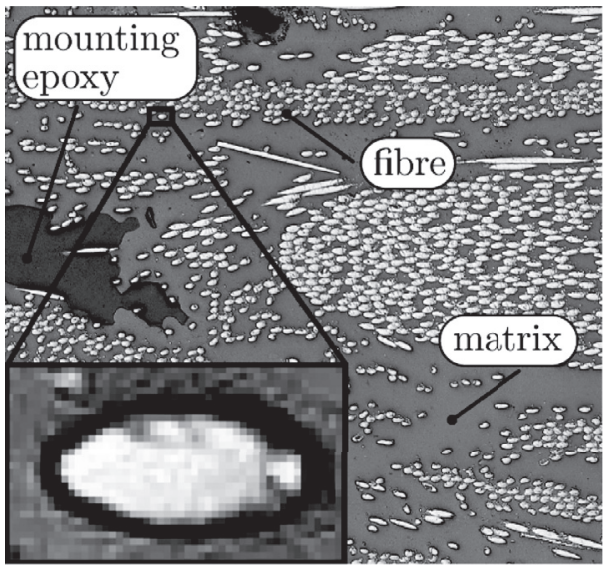

(a)

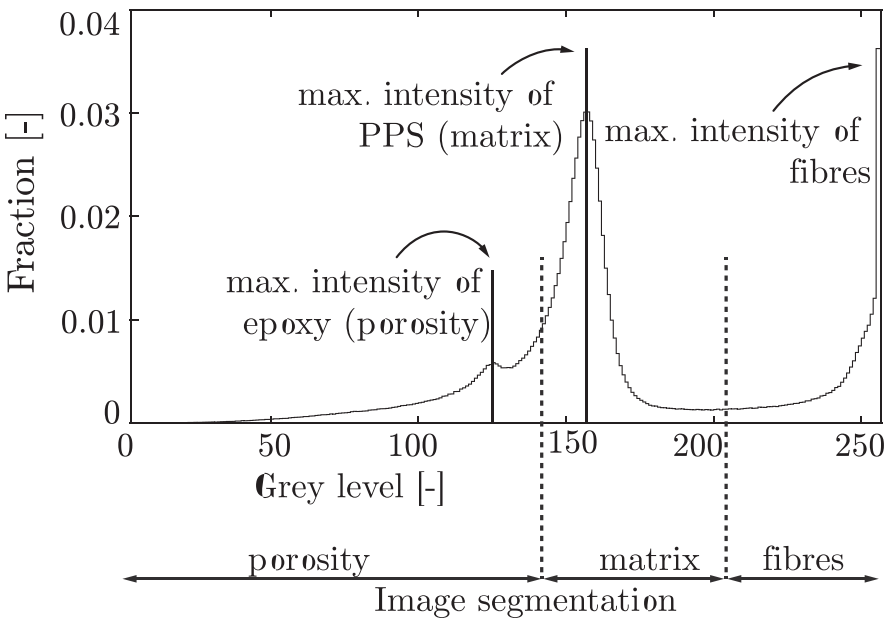

(b)

Fig. B1. Section of a cross-sectional micrograph on the left, showing that fibres, matrix and filled porosities have a different grey level. A close-up of a fibre shows its spurious black envelope The three grey levels are highlighted in the histogram of the image on the right. Automatic segmentation is performed based on the peak locations of the histogram.

would grow proportionally to $\|\overleftrightarrow{\Omega}\|$ (with $\|\overleftrightarrow{\Omega}\|=1$ ). However, for the ellipse centred in $P$, the radius along $\overleftrightarrow{\Omega}$ grows $\left\|\mathbf{T}^{-1} \vec{\Omega}\right\| \|^{-1}$ faster (this expression is $\geq 1$ ). Thus the average growth factors of the two sleeves' radii are proportional to:

$r=\frac{\left\|\mathbf{T}^{-1} \vec{\Omega}\right\|^{-1}+\left\|\mathbf{T}^{\prime-1} \vec{\Omega}\right\|^{-1}}{2}$ with $r \geq 1$. From this, it is straightforward that the ratio $d_{0} / d_{c}$ is the ratio of $r$ over $\|\overleftrightarrow{\Omega}\|$. Here $d_{0}=\left\|\overrightarrow{P P^{\prime}}\right\|$, i.e. the measured distance between $P$ and $P^{\prime}$ in the orthonormal basis $\mathcal{B}_{0}$ of the micrography plane. $d_{c}$ is the corrected centre-to-centre distance mentioned earlier. Hence this ratio is:

$\frac{d_{0}}{d_{c}} \quad=\frac{\left.\left\|\mathbf{T}^{-1} \vec{\Omega}||^{-1}+|| \mathbf{T}^{-1} \vec{\Omega}\right\|\right|^{-1}}{2}$ 
(a)
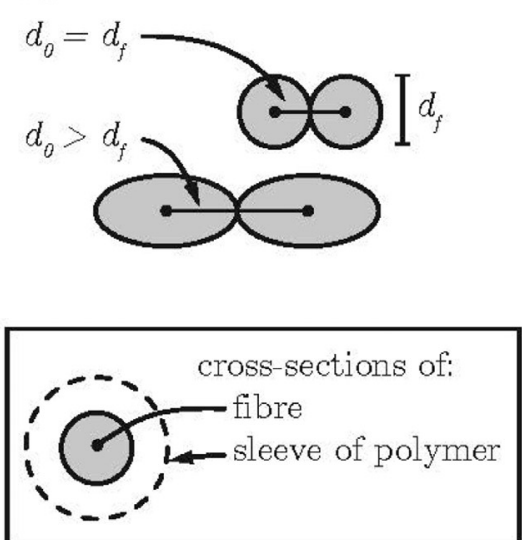

(b)

$$
\begin{aligned}
& d_{0}=2 d_{f} \\
& d_{c}=2 d_{f}
\end{aligned}
$$

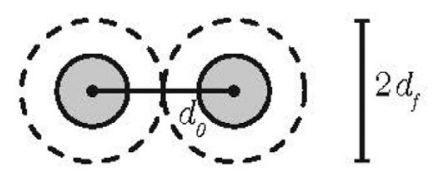

(c)

$$
\begin{aligned}
& d_{0}=3 d_{f} \\
& d_{c}=3 d_{f}
\end{aligned}
$$

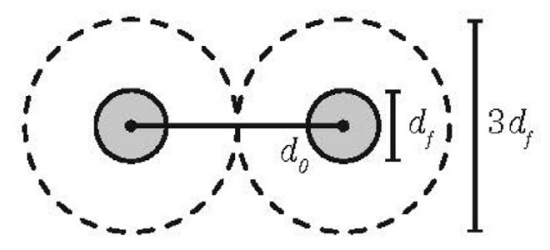

(d)

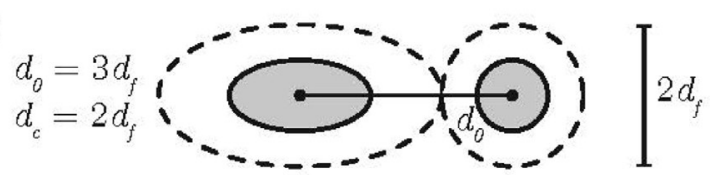

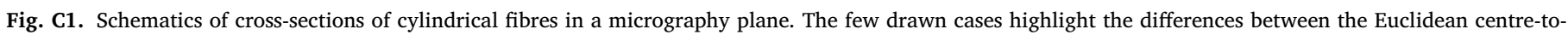
centre distance $d_{0}$ and the true distance between the fibres $d_{c}$ in comparison to the fibre diameter $d_{f}$.

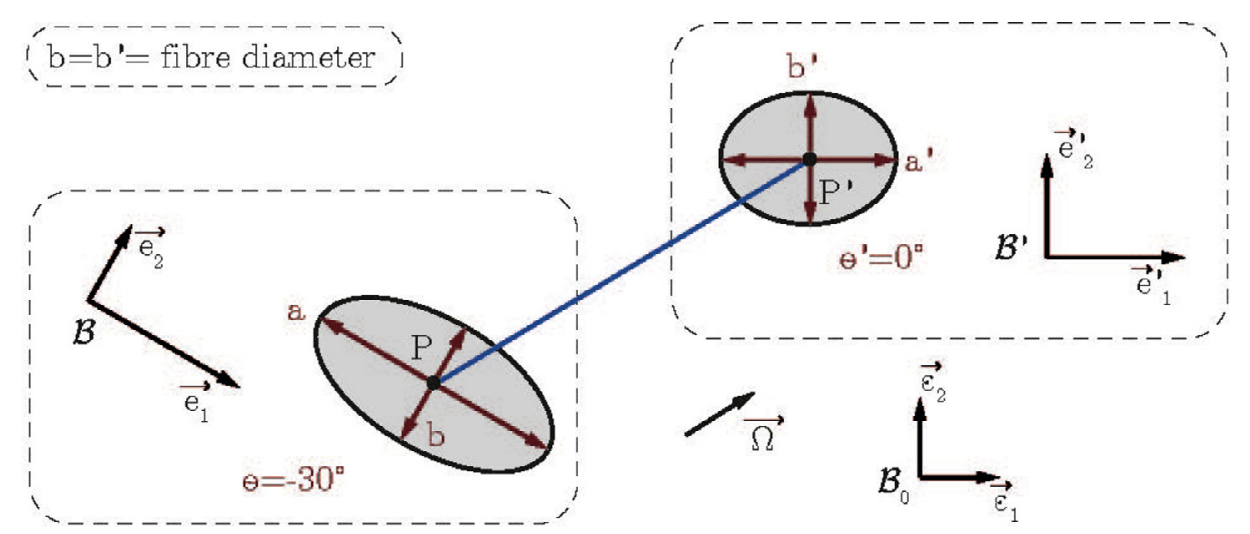

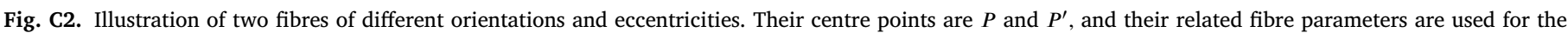

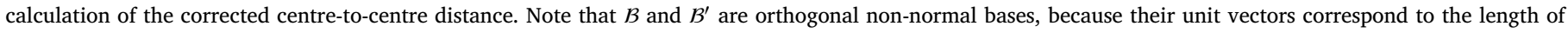
the ellipse axes.

and the expression of $d_{c}$ is:

$d_{c}=\frac{2\left\|\overrightarrow{P P^{\prime}}\right\|}{\left\|\mathbf{T}^{-1} \vec{\Omega}\right\|^{-1}+\left\|\mathbf{T}^{-1} \vec{\Omega}\right\|^{-1}}$

Note that this expression of $d_{c}$ is also the harmonic mean of $\left\|\overrightarrow{P P^{\prime}}\right\| \times$ $\left\|\mathbf{T}^{-1} \vec{\Omega}\right\|$ and $\left\|\overrightarrow{P P^{\prime}}\right\| \times\left\|\mathbf{T}^{\prime-1} \vec{\Omega}\right\|$. This corrected centre-to-centre dis- tance was calculated for all segments of the Delaunay triangulation and used for the refinement.

Appendix D. Overview of image processing steps 


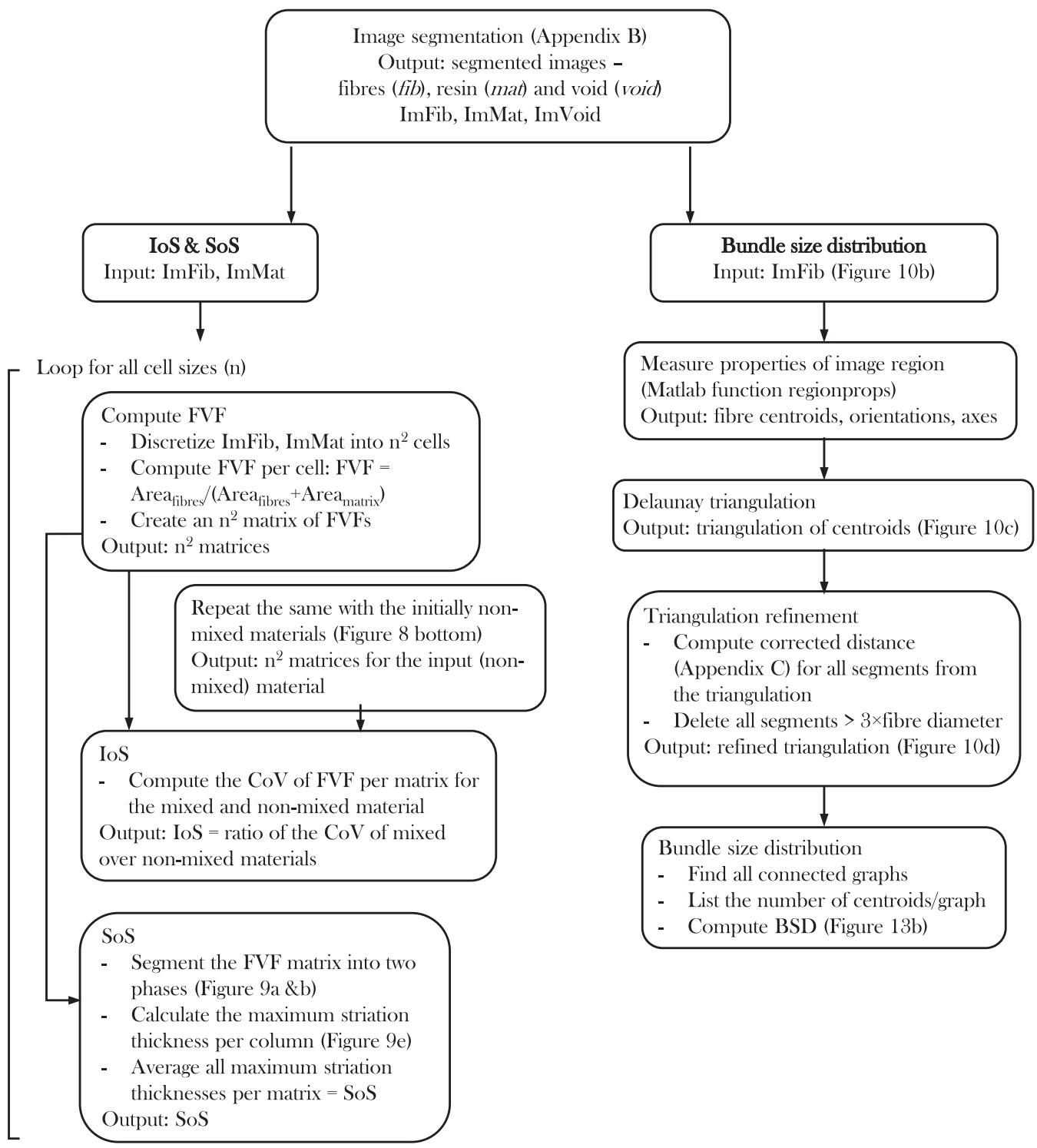

Fig. D1. Schematic overview of the various steps involved towards obtaining the BSD, IoS and SoS from the microscopy figures.

\section{References}

[1] CompositesWorld, Evolution of tailored D-LFT, 2013, https://www.compositesworld.com/articles/evolution-of-tailored-d-lft, [Online; accessed 21-August-2018].

[2] M. Schemme, LFT - development status and perspectives, Reinforc. Plast. 52 (1) (2008), doi:10.1016/S0034-3617(08)70036-5.

[3] G. Tweed, Xycomp ${ }^{\circledR}$ DLF high-performance thermoplastic composite. www.gtweed.com, [Online; accessed 07-November-2018]

[4] T.A. Composites, Compression molded structural components. www.tencatecomposites.com/products/parts-and-services/compression-molded-parts, [Online; accessed 07-November-2018].

[5] W. Schijve, High performance at medium fibre length in long glass fibre polypropylene, Plast., Addit. Compd. 2 (12) (2000) 14-21, doi:10.1016/S1464-391X(00)80121-X.

[6] F.W.J. Van Hattum, S. Van Breugel, LFT: The future of reinforced thermoplastics? Reinforc. Plast. 45 (6) (2001) 42-44, doi:10.1016/S0034-3617(01)80208-3.

[7] T.A. De Bruijn, G.A. Vincent, F.W.J. Van Hattum, Recycling of long fibre thermoplastic composites by low shear mixing, SAMPE Europe, 2016

[8] T.A. De Bruijn, G. Vincent, F.W.J. Van Hattum, Recycling C/PPS laminates into long fibre thermoplastic composites by low shear mixing, in: 21st International Conference in Composite Materials, 2017.

[9] M. Janney, J. Ledger, U. Vaidya, Long fiber thermoplastic composites from recycled carbon fiber, 44th ISTC, 2012.

[10] G. Vincent, T. de Bruijn, S. Wijskamp, M. Adbul Rasheed, R. Akkerman, Shredding and sieving thermoplastic composite scrap: method development analyses of the fibre length distributions, Compos. Part B: Eng. (2019).
[11] L. Mertes, D. Daniel, J. Melack, B. Nelson, L. Martinelli, B. Forsberg, Spatial patterns of hydrology, geomorphology, and vegetation on the floodplain of the amazon river in brazil from a remote sensing perspective, Geomorphology 13 (1995) 215-232, doi:10.1016/0169-555X(95)00038-7.

[12] A. Kukukova, B. Noel, S.M. Kresta, J. Aubin, Impact of sampling method and scale on the measurement of mixing and the coefficient of variance, AIChE J. 54 (504) (2008), doi:10.1002/aic.

[13] A. Yazdanbakhsh, Z. Grasley, B. Tyson, R.K. Abu Al-Rub, Dispersion quantification of inclusions in composites, Compos. Part A: Appl. Sci. Manuf. 42 (1) (2011) 75-83, doi:10.1016/j.compositesa.2010.10.005.

[14] P.V. Danckwerts, The definition and measurement of some characteristics of mixtures, Appl. Sci. Res. Sect. A 3 (4) (1952) 279-296, doi:10.1007/BF03184936.

[15] A. Kukukova, J. Aubin, S.M. Kresta, A new definition of mixing and segregation: Three dimensions of a key process variable, Chem. Eng. Res. Des. 87 (4) (2009) 633-647, doi:10.1016/j.cherd.2009.01.001.

[16] A. Inoue, K. Morita, T. Tanaka, Y. Arao, Y. Sawada, Effect of screw design on fiber breakage and dispersion in injection-molded long glass-fiber-reinforced polypropylene, J. Compos. Mater. 49 (1) (2015) 75-84, doi:10.1177/0021998313514872.

[17] J.E. Spowart, B. Maruyama, D.B. Miracle, Multi-scale characterization of spatially heterogeneous systems: implications for discontinuously reinforced metalmatrix composite microstructures, Mater. Sci. Eng. A 307 (1-2) (2001) 51-66, doi:10.1016/S0921-5093(00)01962-6.

[18] M. Li, V.C. Li, Rheology, fiber dispersion, and robust properties of engineered cementitious composites, Mater. Struct. (2012) 405-420, doi:10.1617/s11527-012-9909-z.

[19] M. Le Baillif, K. Oksman, The effect of processing on fiber dispersion, fiber length, and thermal degradation of bleached sulfite cellulose fiber polypropylene composites, J. Thermoplast. Compos. Mater. 22 (2009) 115-133, doi:10.1177/0892705708091608. 
[20] Z. Wang, J. Gao, T. Ai, W. Jiang, P. Zhao, Quantitative evaluation of carbon fiber dispersion in cement based composites, Constr. Build. Mater. 68 (2014) 26-30, doi:10.1016/j.conbuildmat.2014.06.035.

[21] N. Yang, J. Boselli, I. Sinclair, Simulation and quantitative assessment of homogeneous and inhomogeneous particle distributions in particulate metal matrix composites, J. Microsc. 201 (2) (2001) 189-200, doi:10.1046/j.1365-2818.2001.00766.x.

[22] J. Summerscales, F.J. Guild, N.R.L. Pearce, P.M. Russell, Voronoi cells, fractal dimensions and fibre composites, J. Microsc. 201 (2) (2001) 153-162, doi:10.1046/j.1365-2818.2001.00841.x.

[23] M. Bertram, H. Wendrock, Characterization of planar local arrangement by means of the Delaunay neighbourhood, J. Microsc. 181 (1996) 45-53.

[24] D.J. Bray, S.G. Gilmour, F.J. Guild, T.H. Hsieh, K. Masania, A.C. Taylor, Quantifying nanoparticle dispersion: application of the Delaunay network for objective analysis of sample micrographs, J. Mater. Sci. 46 (19) (2011) 6437-6452, doi:10.1007/s10853-011-5615-4.

[25] D.J. Bray, S.G. Gilmour, F.J. Guild, A.C. Taylor, The effects of particle morphology on the analysis of discrete particle dispersion using Delaunay tessellation, Compos. Part A: Appl. Sci. Manuf. 54 (2013) 37-45, doi:10.1016/j.compositesa.2013.07.003.

[26] B. Bertoncelj, K. Vojisavljević, J. Rihtaršič, G. Trefalt, M. Huskić, E. Žagar, B. Malič, A Voronoi-diagram analysis of the microstructures in bulk-molding compounds and its correlation with the mechanical properties, Express Polym. Lett. 10 (6) (2016) 493-505, doi:10.3144/expresspolymlett.2016.47.

[27] Y. Yang, Methods study on dispersion of fibers in CFRC, Cement Concr. Res. 32 (5) (2002) 747-750, doi:10.1016/S0008-8846(01)00759-1.

[28] N. Ozyurt, T.O. Mason, S.P. Shah, Correlation of fiber dispersion, rheology and mechanical performance of FRCs, Cement Concr. Compos. 29 (2) (2007) 70-79, doi:10.1016/j.cemconcomp.2006.08.006.

[29] W. Yenjaichon, J.R. Grace, C. Jim Lim, C.P. Bennington, Characterisation of gas mixing in water and pulp-suspension flow based on electrical resistance tomography, Chem. Eng. J. 214 (2013) 285-297, doi:10.1016/j.cej.2012.10.057.
[30] T. Mattfeldt, Explorative statistical analysis of planar point processes, J. Microsc. 220 (2005) 131-139.

[31] L. Pernenkil, C.L. Cooney, A review on the continuous blending of powders, Chem. Eng. Sci. 61 (2) (2006) 720-742, doi:10.1016/j.ces.2005.06.016.

[32] G. Vincent, T. de Bruijn, M.I. Abdul Rasheed, S. Wijskamp, R. Akkerman, Fibre length distribution of shredded thermoplastic composite scrap, in: 21th International Conference on Composite Materials, 2017.

[33] M.I. Abdul Rasheed, Compression molding of chopped woven thermoplastic composite flakes: a study on processing and performance, University of Twente, 2016 Ph.D. thesis.

[34] A. Beukers, F. Wiltink, J. Van Breugel, Device and method for the preparation of a mixture comprisong fibre-reinforced thermoplastics plastics pellets, 2000. Patent: WO 000002718.

[35] G. Vincent, T. de Bruijn, S. Wijskamp, M. Van Drongelen, R. Akkerman, Process- and material-induced heterogeneities in recycled thermoplastic composites, J. Thermoplast. Comp. Mater. (2020), doi:10.1177/0892705720979347.

[36] C. Servais, A. Luciani, J.-A.E. Månson, Fiberfiber interaction in concentrated suspensions: dispersed fiber bundles, J. Rheol. 43 (4) (1999), doi:10.1122/1.551014.

[37] C. Servais, J.-A.E. Månson, S. Toll, Fiberfiber interaction in concentrated suspensions: dispersed fiber, J. Rheol. 43 (4) (1999), doi:10.1122/1.551014.

[38] J.L. Thomason, The influence of fibre length and concentration on the properties of glass fibre reinforced polypropylene: 5. Injection moulded long and short fibre PP, Comp. Part A: Appl. Sci. Manuf. 33 (12) (2002) 1641-1652, doi:10.1016/S1359-835X(02)00179-3.

[39] H. Meijer, M. Singh, P. Anderson, On the performance of static mixers: a quantitative comparison, Progr. Polym. Sci. 37 (10) (2012) 1333-1349, doi:10.1016/j.progpolymsci.2011.12.004. 